\title{
PROBABLE HIGH GROUND-WATER LEVELS \\ ON CAPE COD, MASSACHUSETTS
}

U.S. GEOLOGICAL SURVEY

Water-Resources Investigations

Open-File Report 80-1008

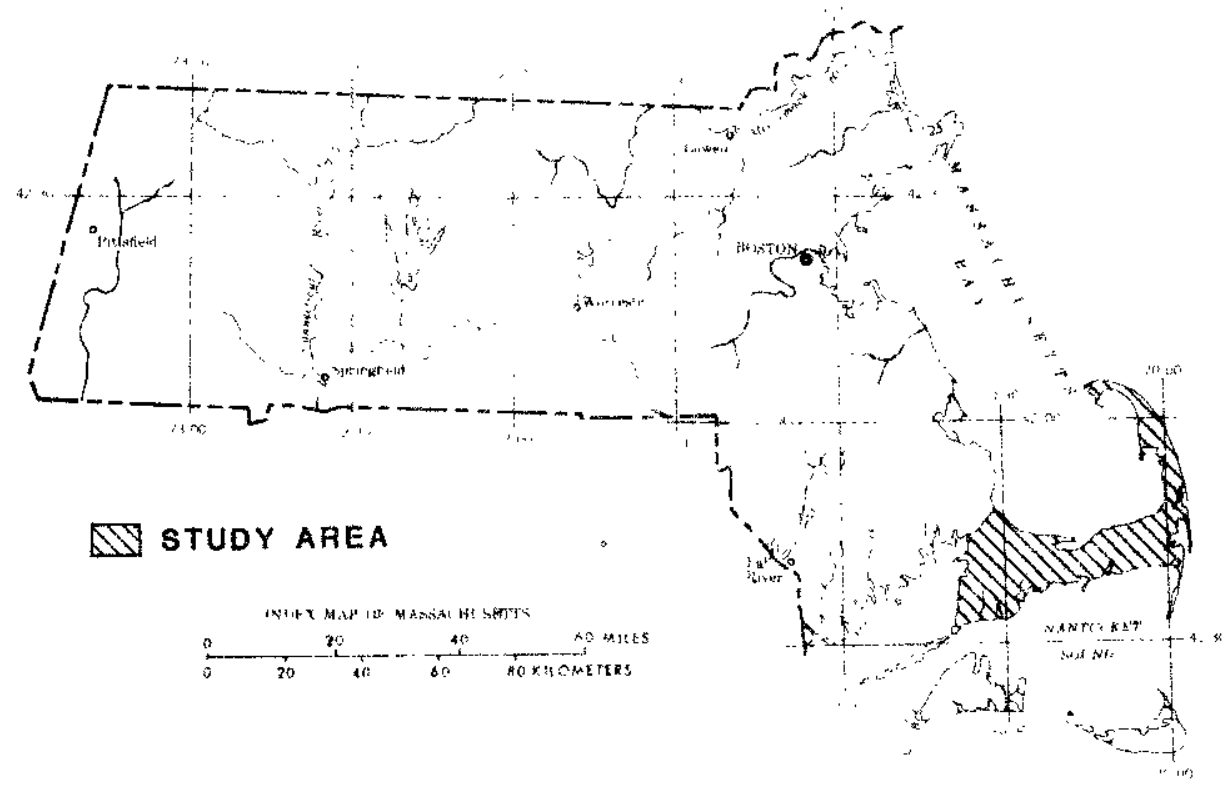

Prepared in cooperation with the COMMONWEALTH OF MASSACHUSETTS DEPARTMENT OF ENVIRONMENTAL. QUALITY ENGINEERING

$$
\begin{aligned}
& R+D \\
& F i l e \text { Copy } \\
& T S B \\
& \text { westbonsh }
\end{aligned}
$$


PROBABLE HIGH GR̈OUND-WATER LEVELS ON CAPE COD, MASSACHUSETTS

By Michael H. Frimpter

\section{U.S. GEOLOGICAL SURVEY}

Water-Resources Investigations

Open-File Report 80-1008

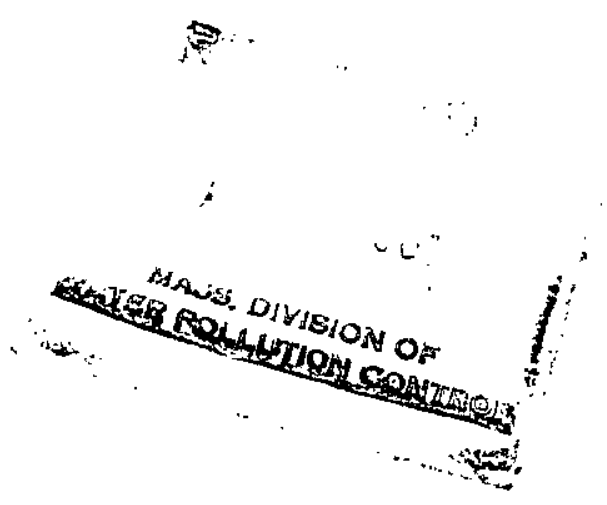

Prepared in cooperation with the

COMMONWEALTH OF MASSACHUSETTS

DEPARTMENT OF ENVIRONMENTAL QUALITY ENGINEERING

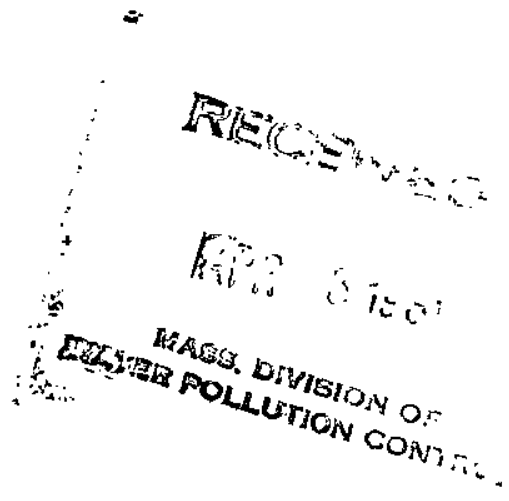




\section{UNITED STATES DEPARTMENT OF THE INTERIOR \\ CECIL D. ANDRUS, Secretary \\ GEOLOGICAL SURVEY \\ H. William Menard, Director}

For additional information write to:

U.S. Geological Survey

150 Causeway Street, Suite 1001

Boston, MA 02114 
Abstract-

Introduction-

Ground-water-level fluctuations

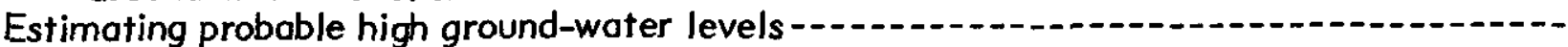

Procedure of estimating the probable high water level -..-...-

An example -

Summary and conclusions -

Selected bibliography-1.-.

\section{ILLUSTRATIONS}

(Plates are in pocket.)

Plate I. Annual water-level ranges and index wells, west part of Cape Cod.

2. Annual water-level ranges and index wells, east part of Cape Cod.

3. Flow diagram of estimating procedure for Cape Cod.

4. Flow-diagram example.

Figure 1: Seasonal water-level fluctuations

2. Long-term water-level fluctuations

3. Frequency, by month, of maximum annual water level in 13 observation wells on Cape Cod -...... 5

4. Correlation of water levels in two observation wells

5. Difference between observed and estimated highest water levels at 49 sites

\section{TABLES}

Table I. Table of woter-level adjustments, in feet, for use with index well:

2. Barnstable AIW-230 Barnstable AIW-247

3. Bourne BHW-198

Brewster BMW-21

5. Chatham CGW-138

Sandwich SDW-252

7. Sandwich SDW-253

8. Truro TSW-89

9. Wellfleet WNW-17-14

10. Example of U.S. Geological Survey monthly summary of ground-water levels in Massachusetts -

CONVERTING INCH-POUND UNITS TO INTERNATIONAL SYSTEM UNITS (SI).

The following factors may be used to convert the inch-pound units published herein to the International System Units (SI):

Inch-pound units

Foot (ft)
Multiply by

0.3048
To obtain SI Units

meter $(m)$ 
PROBABLE HIGH GROUND-WATER LEVELS ON CAPE COD, MASSACHUSETTS

By Michael H. Frimpter

\begin{abstract}
Water-level records from 146 short-term (I year) observation wells and 13 long-term (16-28 years) observation wells were used to estimate the probable high ground-water level that could occur at any site on Cape Cod. The estimation was based on correlation of a single water-level measurement from a test site with water-level records of the nine index wells. Maps showing areas of Cape Cod represented by the nine index wells and showing five ranges of water-level fluctuation are used in conjunction with tables of water-level adjustments to make the estimates.
\end{abstract}

\title{
INTRODUCTION
}

This report describes how the high ground-water level that would probably occur at any site on Cape Cod could be estimated from one water-level measurement at that site. It is the first product of a study in cooperation with the Massachusetts Department of Environmental Quality Engineering to evaluate the use of records of ground-water levels and observation-well networks constructed in water-table aquifers for estimating probable high ground-water levels at any site in the State.

The probable high ground-water levels are needed to apply State regulations governing construction of septic systems. The regulations contained in "Commonwealth of Massachusetts, Department of Environmental Quality Engineering, the State Environmental Code, Minimum Requirements for the Subsurface Disposal of Sanitary Sewage, 1977, Title 5" require that "Leaching fields shall not be constructed in areas where the maximum ground-water elevation is less than 4 feet below the bottom of the field." The State Environmental .Code, Title 5, defines the "maximum ground-water elevation" as the "...height of the ground-water table when it is at its maximum level...". In this report, "maximum ground-water level" is taken to be the highest level (elevation) of the water table which is also the minimum depth below land surface as illustrated in figure $\mathrm{I}$.

Ground-water levels in Massachusetts rise and fall several feet each year. The amount of annual fluctuation differs from place to place, depending on topographic position, hydrologic characteristics of aquifers, and climate. A single random water-level measurement at a site is, therefore, unlikely to represent either the highest or lowest level at that site. Although the State Code suggests that allowances should be made for measurements made when the water level is not at a high level, there have been no scientific guidelines for making such allowances.

\section{Ground-Water-Level Fluctuations}

The annual cyclic rise and fall of the water table under natural conditions in Massachusetts is due to seasonal differences in the rate of ground-water recharge and relatively constant discharge. Precipitation is fairly evenly distributed from month to month throughout each year but there is little or no ground-water recharge from precipitation during the late spring to early fall growing season (a period of about 180 days each year), when evapotranspiration rates are high. Therefore, the water table generally declines steadily during the growing season, except during periods of much precipitation, such as during hurricanes. Ground-water bodies are recharged most during late winter or early spring both from precipitation and melting of snow and ice, when annual ground-water levels (fig. I) generally reach their maximum. 


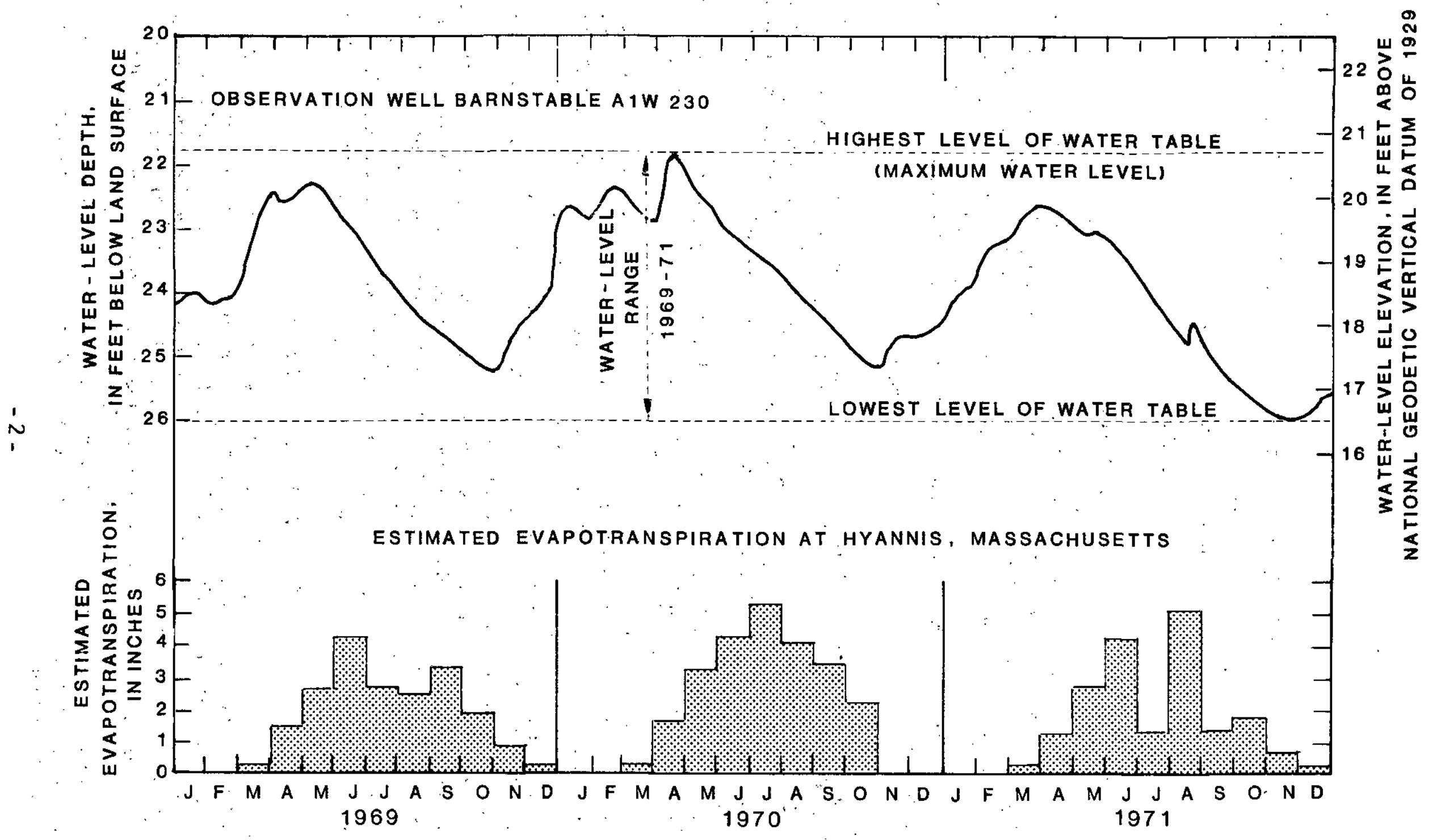

Figure 1.--Seasonal water-level fluctuations 
Differences in water levels from year to year are largely due to the amount and distribution of annual precipitation (fig. 2). Long-term ground-water-level fluctuations result from annual precipitation differences, and annual fluctuations result from seasonal variations in evapotranspiration, which is fairly constant from year to year.

Proximity to major pumping centers and tidal waters can also affect the time of occurrence of maximum annual water levels. For example, the water level most frequently reaches an annual maximum in January in well TSW-! in Truro, which is in a pumping center. Additionally, water levels in two wells are affected by tidal fluctuations in Cape Cod Bay or Cape Cod Canal.

Long-term (more than 13 years) records of water levels from 13 observation wells and short-term ( 1 year) records from 146 observation wells were used in this analysis.

Water-level records for the 13 observation wells on Cape Cod range from 16 to 28 years in length and include more than 2,600 monthly measurements. More than 57 percent of the maximum annual water levels occurred in March, April, and May--30percent in April alone (fig. 3). Water level was at an annual maximum most frequently in April in 10 of the 13 wells and in January, March, and May in the remaining three.

The greatest fluctuation in the 13 long-term-observation wells is 9.27 feet in SDW 253 in Sandwich. The median recorded high water-level fluctuation for the 13 wells is 5.09 feet. In these long-term-observation wells, the maximum fluctuation in any I year was 4.26 feet in 1966 in well BHW 198 in Bourne.

Generally, the greater the depth to the water table below land surface, the later the water level reaches the annual maximum. For example, in observation well SDW-253 in Sandwich, the mean depth to water is more than 20 feet deeper ( 49.8 feet below land surface) than in most other observation wells, and the water level reaches an annual maximum most frequently in May, I month later than in the other wells.

Water-level data from both the long- and short-term observation wells indicate that, generally, fluctuations are least near constant-head boundaries, such as the ocean, sounds, bays, estuaries, and salt marshes. For example, the water level in well TSW-89 in Truro fluctuated only 2.60 feet from 1963 to 1978. Exceptions to this observation are diurnal fluctuations in wells within 300 feet of tidal water bodies having large tidal ranges such as Cape Cod Bay (10 feet). At a distance of more than 300 feet from the shore, water-table fluctuations caused by tides are dampened to less than 2 feet.

\section{ESTIMATING PROBABLE HIGH GROUND-WATER LEVELS}

Estimates of potential water-level rise from a current level at an observation well can be made from the historical water-level records for that well. Such estimates are based on the assumption that the stresses which cause water-level changes will be the same as those that controlled the changes over the historical record. Generally, the longer the period of historical record, the less likely are the estimates of high water levels to be exceeded. Additionally, the reliability of the estimates may. be improved if the historical record is representative of a longer period of these variable conditions which control water levels, such as annual precipitation.

Water-level observations from the 13 long-term wells on Cape Cod span the period in which the record-low ground-water levels of the mid-sixties drought and the record-high levels of the late sixties and early seventies of high precipitation were recorded. Of six observation wells throughout Massachusetts with more than 39 years of record, water levels in four reached their highest levels and in three wells reached their lowest levels during the period over which the Cape Cod wells were observed. Therefore, the water-level records of all 13 long-term-observation wells on Cape Cod probably show the greatest water-level fluctuations on the Cape for the 39-year period of record.

Records of annual precipitation collected at Hyannis since 1892 show that the greatest annual precipitation was 61.91 inches in 1972 and the smallest was 27.97 inches in 1965 . Recharge to ground-water bodies on Cape Cod depends on precipitation, and although the records of the observation wells span a minimum period of 16 years, those 16 years contain the years of maximum and minimum annual preciptation recorded for Hyannis, Massachusetts, over the last 86 years. Although distribution of annual precipitation throughout the year will also have some influence on the maximum altitude to which ground-water will rise, it seems likely that water-level ranges recorded for these 13 observation wells probably are representative of the 86-year period. 


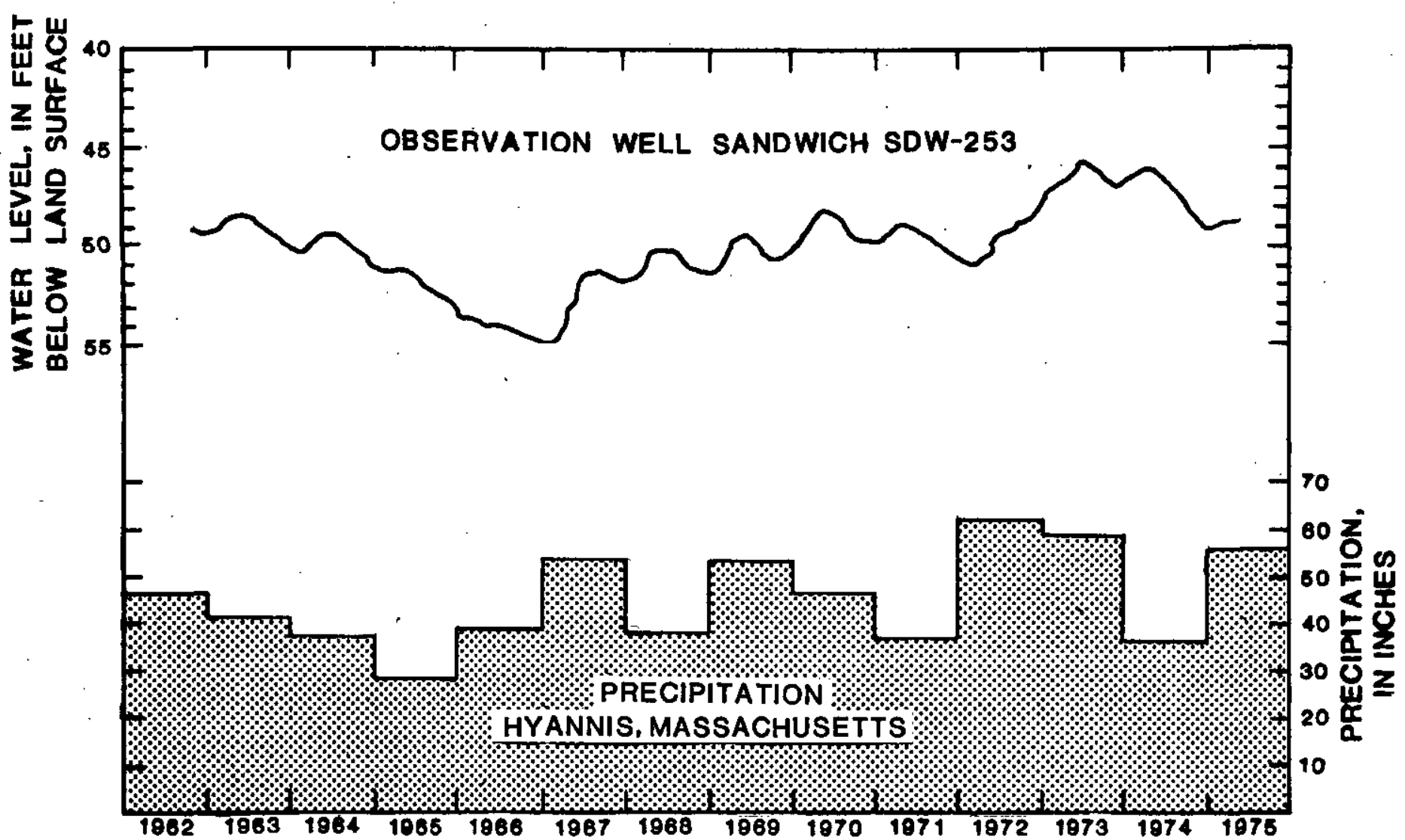

Fi.gure 2.--Long-term water-level fluctuations 


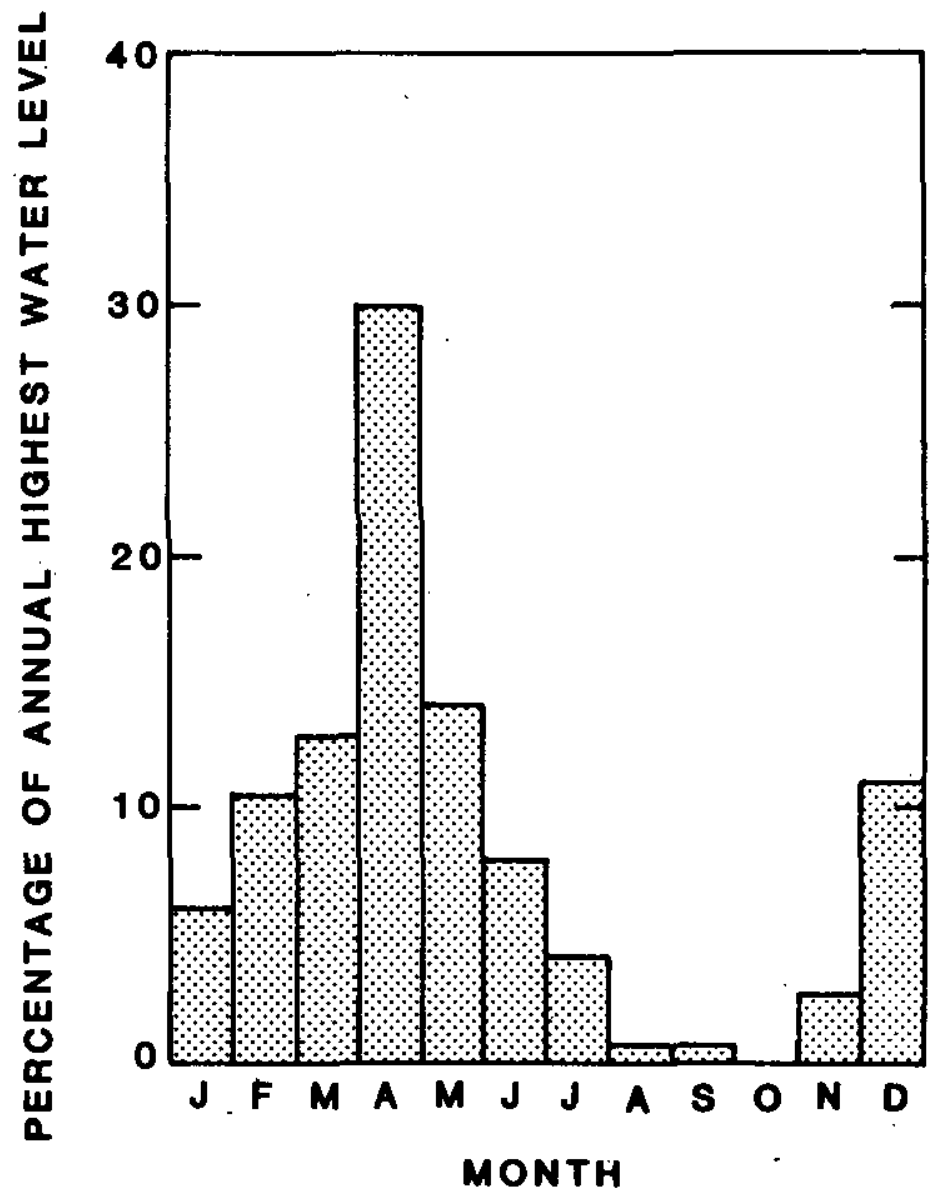

Figure 3.-- Frequency, by month, of maximum annual water level in 13 observation wells on Cape Cod 
An estimate of the probable high water level at a site might be made on the basis of the assumption that water-level fluctuations at the site are directly correlated with water-level fluctuations at a selected observation well named as an index well. The mathematical expression of this assumption follows:

$$
\frac{S_{c}-S_{h}}{O W_{c}-O W_{\max }}=\frac{S_{r}}{O W_{r}}
$$

where: $\mathrm{S}_{\mathrm{C}} \quad=$ measured depth to water at the site;

$\mathrm{S}_{\mathrm{h}} \quad=$ estimated depth to probable high water level at the site;

$\mathrm{OW}_{\mathrm{C}}=$ measured depth to water in the index well which is used to correlate with the water levels at the site;

$O W_{\text {max }}=$ depth to recorded maximum water level at the index well which is used to correlate with the water levels at the site;

$\mathrm{S}_{\mathbf{r}}=$ recorded upper limit of the annual range of water level in a particular zone where the site is located. The values of the recorded upper limit of the ranges are shown in plates $I$ and 2 . For example, if the site is in zone A, $S_{r}=2$ feet;

$\mathrm{OW}_{\mathrm{r}}=$ recorded upper limit of annual range of water level at the index well which is used to correlate with the water levels at the site.

Rearranging the equation, the estimated depth to probable high water level at the site $\left(S_{h}\right)$ is given by:

$$
S_{h}=S_{c}-\frac{S_{r}}{O W_{r}}\left(O W_{c}-O W_{\max }\right)
$$

In the above equation, $\mathrm{S}_{\mathrm{c}}$ and $\mathrm{OW}_{\mathrm{c}}$ are measured at the time of interest. $O W_{\max }$ is a constant and obtained from the long-term records of each index well; the value of $O W_{\text {max }}$ for each index well is shown by the first columns of tables $1-9 . S_{r}$ and $O W_{r}$ are obtained from plates $I$ and 2. For example, if the site is located in zone $A$, the upper limit of annual range of water level in zone $A$ is 2 feet (plates $I$ and 2 ), then $S_{r}=2$ feet. If the correlated index well is AIW-230 which is located in zone C where the upper limit of annual range of water level is 4 feet (plates I and 2), then the recorded upper limit of annual range of water level in index well $\mathrm{AIW}-230$ is also $4 \mathrm{feet}$, therefore, $\mathrm{OW}_{\mathrm{r}}=4$ feet.

Magnitude of ground-water fluctuation for an area may be estimated from fluctuations observed in an observation well, provided that hydrologic conditions at the observation well are representative of the area. Most of Cape Cod consists of uniform glacial outwash deposits of sand and gravel, or of sandy glacial moraine. Hydraulic conductivities and storage coefficients of these deposits are relatively uniform, as demonstrated by the smooth shield-shaped water table that is developed in five separate freshwater aquifers beneath the Cape (LeBlanc and Guswa, 1977) in response to recharge from precipitation. Areas of till along Buzzards Bay have been excluded from the estimation because of different geohydrologic properties.

As an aid to the estimating procedure, Cape Cod is subdivided into nine areas in which water-level fluctuations in each area are best represented by an index well (plates 1 and 2) selected from the 13 long-term-observation wells. Wells TSW-I, BMW-22, FSW-5, and MIW-13 were not used as index wells. The areas were mapped on a basis of geographic proximity, geohydrologic properties of the drift deposits, location with respect to hydrologic boundaries, and by correlating the October 1975 to March 1977 water levels in 146 short-term observation wells with the water levels in the 13 long-term observation wells for the same period. 
Table 1:--Water-level adjustments, in feet, for use with index well Barnstable AIW-230 (located in zone C)

\begin{tabular}{|c|c|c|c|c|c|c|}
\hline $\begin{array}{l}\text { Measured water } \\
\text { leve } 1 \text { (ow }) \\
\text { (in feet below } \\
\text { 1 and surface) }\end{array}$ & $\frac{\text { Zone A }}{(2 / 4)^{1 /}}$ & $\frac{\text { Zone B }}{(3 / 4) \underline{1} /}$ & $\frac{\text { Zone } C}{(4 / 4) \underline{1} /}$ & $\cdots$ & $\frac{\text { Zone D }}{(5 / 4) \underline{1 /}}$ & $\frac{\text { Zone E }}{(6 / 4) \underline{1}}$ \\
\hline $\begin{array}{l}21.12 / \\
21.2 \\
21.3 \\
21.4 \\
21.5 \\
21.6 \\
21.7 \\
21.8 \\
21.9 \\
22.0 \\
22.1 \\
22.2 \\
22.3 \\
22.4 \\
22.5 \\
22.6 \\
22.7 \\
22.8 \\
22.9 \\
23.0 \\
23.1 \\
23.2 \\
23.3 \\
23.4 \\
23.5 \\
23.6 \\
23.7 \\
23.8 \\
23.9 \\
24.0 \\
24.1 \\
24.2 \\
24.3 \\
24.4 \\
24.5 \\
24.6 \\
24.7 \\
24.8 \\
24.9 \\
25.0 \\
25.1 \\
25.2 \\
25.3 \\
25.4 \\
25.5 \\
25.6 \\
25.7 \\
25.8 \\
25.9 \\
26.0 \\
26.1 \\
26.2 \\
26.3 \\
26.4 \\
26.5 \\
26.6 \\
26.7 \\
.\end{array}$ & $\begin{array}{l}0.0 \\
.0 \\
.1 \\
.1 \\
.2 \\
.2 \\
.3 \\
.3 \\
.4 \\
.4 \\
.5 \\
.5 \\
.6 \\
.6 \\
.7 \\
.7 \\
.8 \\
.8 \\
.9 \\
.9 \\
1.0 \\
1.0 \\
1.1 \\
1.1 \\
1.2 \\
1.2 \\
1.3 \\
1.3 \\
1.4 \\
1.4 \\
1.5 \\
1.5\end{array}$ & $\begin{array}{r}0.0 \\
.1 \\
.1 \\
.2 \\
.3 \\
.4 \\
.4 \\
.5 \\
.6 \\
.7 \\
.7 \\
.8 \\
.9 \\
1.0 \\
1.0 \\
1.1 \\
1.2 \\
1.3 \\
1.3 \\
1.4 \\
1.5 \\
1.6 \\
1.6 \\
1.7 \\
1.8 \\
1.9 \\
1.9 \\
2.0 \\
2.1 \\
2.2 \\
2.2 \\
2.3 \\
2.4 \\
2.5 \\
2.5 \\
2.6 \\
2.7 \\
2.8 \\
2.8 \\
2.9 \\
3.0 \\
3.1 \\
3.1 \\
3.2 \\
3.3 \\
3.4 \\
3.4 \\
3.5 \\
3.6 \\
3.7 \\
3.7 \\
3.8 \\
3.9 \\
4.0 \\
4.0 \\
4.1 \\
4.2\end{array}$ & 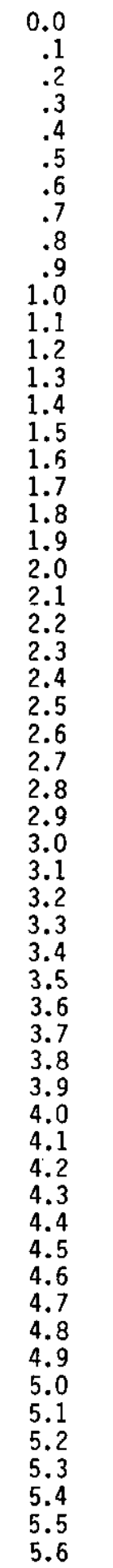 & $\begin{array}{l}. \\
\vdots \\
\cdot \\
\cdot \\
\cdot \\
\cdot\end{array}$ & $\begin{array}{l}0.0 \\
.1 \\
.2 \\
.4 \\
.5 \\
.6 \\
.7 \\
.9 \\
1.0 \\
1.1 \\
1.2 \\
1.4 \\
1.5 \\
1.6 \\
1.7 \\
1.9 \\
2.0 \\
2.1 \\
2.2 \\
2.4 \\
2.5 \\
2.6 \\
2.7 \\
2.9 \\
3.0 \\
3.1 \\
3.2 \\
3.4 \\
3.5 \\
3.6 \\
3.7 \\
3\end{array}$ & 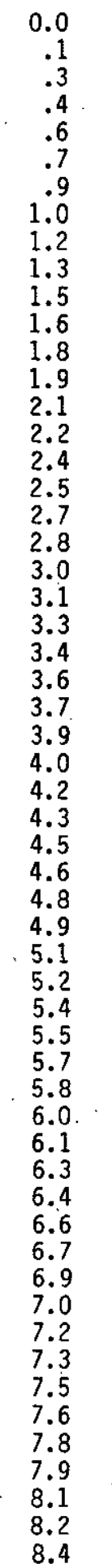 \\
\hline
\end{tabular}

$\underline{1} / \mathrm{S}_{r} / 0 \mathrm{Wr}_{r}=$

2/Recorded highest water level $\left(0 W_{\max }\right)$. 
Table 2.--Water-level adjustments, in feet, for use with index well Barnstable AlW-247 (located in zone B)

\begin{tabular}{|c|c|c|c|c|}
\hline $\begin{array}{l}\text { Measured water } \\
\text { level (owc) } \\
\text { (in feet below } \\
\text { land surface) }\end{array}$ & $\frac{\text { Zone A }}{(2 / 3) \underline{1}}$ & $\frac{\text { Zone B }}{(3 / 3) \underline{1 /}}$ & $\frac{\text { Zone C }}{(4 / 3) \underline{1} /}$ & $\frac{\text { Zone D. }}{(5 / 3)^{1 /}}$ \\
\hline $\begin{array}{l}21.02 \\
21.1 \\
21.2 \\
21.3 \\
21.4 \\
21.5 \\
21.6 \\
21.7 \\
21.8 \\
21.9 \\
22.0 \\
22.1 \\
22.2 \\
22.3 \\
22.4 \\
22.5 \\
22.6 \\
22.7 \\
22.8 \\
22.9 \\
23.0 \\
23.1 \\
23.2 \\
23.3 \\
23.4 \\
23.5 \\
23.6 \\
23.7 \\
23.8 \\
23.9 \\
24.0 \\
24.1 \\
24.2 \\
24.3 \\
24.4 \\
24.5 \\
24.6 \\
24.7 \\
24.8 \\
24.9 \\
25.0 \\
25.1 \\
25.2 \\
25.3 \\
25.4 \\
25.5 \\
25.6 \\
25.7 \\
25.8 \\
25.9 \\
26.0 \\
26.1 \\
26.2 \\
26.3\end{array}$ & $\begin{array}{l}0.0 \\
.1 \\
.1 \\
.2 \\
.3 \\
.3 \\
.5 \\
.5 \\
.6 \\
.7 \\
.7 \\
.8 \\
.9 \\
.9 \\
1.0 \\
1.1 \\
1.1 \\
1.2 \\
1.3 \\
1.3 \\
1.4 \\
1.5 \\
1.5 \\
1.6 \\
1.7 \\
1.7 \\
1.8 \\
1.9 \\
1.9 \\
2.0 \\
2.1 \\
2.1 \\
2.2 \\
2.3 \\
2.3 \\
2.4 \\
2.5 \\
2.5 \\
2.6 \\
2.7 \\
2.7 \\
2.8 \\
2.9 \\
2.9 \\
3.0 \\
3.1 \\
3.1 \\
3.2 \\
3.3 \\
3.3 \\
3.4 \\
3.5 \\
3.6\end{array}$ & $\begin{array}{r}0.0 \\
.1 \\
.2 \\
.3 \\
.4 \\
.5 \\
.6 \\
.7 \\
.8 \\
.9 \\
.9 \\
1.0 \\
1.1 \\
1.2 \\
1.3 \\
1.4 \\
1.5 \\
1.6 \\
1.7 \\
1.8 \\
1.9 \\
2.0 \\
2.1 \\
2.2 \\
2.3 \\
2.4 \\
2.5 \\
2.6 \\
2.7 \\
2.8 \\
2.9 \\
3.0 \\
3.1 \\
3.2 \\
3.3 \\
3.4 \\
3.5 \\
3.6 \\
3.7 \\
3.8 \\
3.9 \\
4.0 \\
4.1 \\
4.2 \\
.4 .3 \\
4.4 \\
4.5 \\
4.6 \\
4.7 \\
4.8 \\
4.9 \\
5.0 \\
5.1 \\
5.2 \\
5.3 \\
\end{array}$ & $\begin{array}{r}0.0 \\
.1 \\
.3 \\
.4 \\
.5 \\
.7 \\
.8 \\
.9 \\
1.1 \\
1.2 \\
1.3 \\
1.5 \\
1.6 \\
1.7 \\
1.9 \\
2.0 \\
2.1 \\
2.3 \\
2.4 \\
2.5 \\
2.7 \\
2.8 \\
2.9 \\
3.1 \\
3.2 \\
3.3 \\
3.5 \\
3.6 \\
3.7 \\
3.9 \\
4.0 \\
4.1 \\
4.3 \\
4.4 \\
4.5 \\
4.7 \\
4.8 \\
4.9 \\
5.1 \\
5.2 \\
5.3 \\
5.5 \\
5.6 \\
5.7 \\
5.9 \\
6.0 \\
6.1 \\
6.3 \\
6.4 \\
6.5 \\
6.6 \\
6.8 \\
6.9 \\
7.0\end{array}$ & $\begin{array}{r}0.0 \\
.2 \\
.3 \\
.5 \\
.7 \\
.8 \\
1.0 \\
1.2 \\
1.3 \\
1.5 \\
1.7 \\
1.8 \\
2.0 \\
2.2 \\
2.3 \\
2.5 \\
2.7 \\
2.8 \\
3.0 \\
3.2 \\
3.3 \\
3.5 \\
3.7 \\
3.8 \\
4.0 \\
4.2 \\
4.3 \\
4.5 \\
4.7 \\
4.8 \\
5.0 \\
5.2 \\
5.3 \\
5.5 \\
5.7 \\
5.8 \\
6.0 \\
6.2 \\
6.3 \\
6.5 \\
6.7 \\
6.8 \\
7.0 \\
7.2 \\
7.3 \\
7.5 \\
7.7 \\
7.8 \\
8.0 \\
8.2 \\
8.3 \\
8.5 \\
8.7 \\
8.9\end{array}$ \\
\hline
\end{tabular}

$\underline{1 / \mathrm{S}_{\mathrm{r}}} / 0 \mathrm{~W}_{\mathrm{r}}=$

2/Recorded highest water level $\left(O W_{\max }\right)$. 
Table 3.--Water-level adjustments, in feet, for use with index well Bourne BHW-198 (located in zone B)

\begin{tabular}{|c|c|c|c|c|c|c|c|}
\hline $\begin{array}{l}\text { Measured water } \\
\text { level (OW }) \\
\text { (in feet below } \\
\text { land surface) }\end{array}$ & $\frac{\text { Zone A }}{(2 / 3) \underline{1} /}$ & $\frac{\text { Zone B }}{(3 / 3) \underline{1 /}}$ & $\frac{\text { Zone C }}{(4 / 3) \underline{1} /}$ & $\begin{array}{l}\text { Measured water } \\
\text { level (OW }) \\
\text { (in feet below } \\
\text { land surface) }\end{array}$ & $\frac{\text { Zone A }}{(2 / 3) \underline{1} /}$ & $\frac{\text { Zone B }}{(3 / 3) \underline{1 /}}$ & $\frac{\text { Zone C }}{(4 / 3) \underline{I}}$ \\
\hline $\begin{array}{l}30.42 / \\
30.5 \\
30.6 \\
30.7 \\
30.8 \\
30.9 \\
31.0 \\
31.1 \\
31.2 \\
31.3 \\
31.4 \\
31.5 \\
31.6 \\
31.7 \\
31.8 \\
31.9 \\
32.0 \\
32.1 \\
32.2 \\
32.3 \\
32.4 \\
32.5 \\
32.6 \\
32.7 \\
32.8 \\
32.9 \\
33.0 \\
33.1 \\
33.2 \\
33.3\end{array}$ & $\begin{array}{l}0.0 \\
.1 \\
.1 \\
.2 \\
.3 \\
.3 \\
.4 \\
.5 \\
.5 \\
.6 \\
.7 \\
.7 \\
.8 \\
.9 \\
.9 \\
1.0 \\
1.1 \\
1.1 \\
1.2 \\
1.3 \\
1.3 \\
1.4 \\
1.5 \\
1.5 \\
1.6 \\
1.7 \\
1.7 \\
1.8 \\
1.9 \\
1.9\end{array}$ & $\begin{array}{r}0.0 \\
.1 \\
.2 \\
.3 \\
.4 \\
.5 \\
.6 \\
.7 \\
.8 \\
.9 \\
1.0 \\
1.1 \\
1.2 \\
1.3 \\
1.4 \\
1.5 \\
1.6 \\
1.7 \\
1.8 \\
1.9 \\
2.0 \\
2.1 \\
2.2 \\
2.3 \\
2.4 \\
2.5 \\
2.6 \\
2.7 \\
2.8 \\
2.9\end{array}$ & $\begin{array}{l}0.0 \\
.1 \\
.3 \\
.4 \\
.5 \\
.7 \\
.8 \\
.9 \\
1.1 \\
1.2 \\
1.3 \\
1.5 \\
1.6 \\
1.7 \\
1.9 \\
2.0 \\
2.1 \\
2.3 \\
2.4 \\
2.5 \\
2.7 \\
2.8 \\
2.9 \\
3.1 \\
3.2 \\
3.3 \\
3.5 \\
3.6 \\
3.7 \\
3.9\end{array}$ & $\begin{array}{l}33.4 \\
33.5 \\
33.6 \\
33.7 \\
33.8 \\
33.9 \\
34.0 \\
34.1 \\
34.2 \\
34.3 \\
34.4 \\
34.5 \\
34.6 \\
34.7 \\
34.8 \\
34.9 \\
35.0 \\
35.1 \\
35.2 \\
35.3 \\
35.4 \\
35.5 \\
35.6 \\
35.7 \\
35.8 \\
35.9 \\
36.0 \\
36.1 \\
36.2\end{array}$ & $\begin{array}{l}2.0 \\
2.1 \\
2.1 \\
2.2 \\
2.3 \\
2.3 \\
2.4 \\
2.5 \\
2.5 \\
2.6 \\
2.7 \\
2.7 \\
2.8 \\
2.9 \\
2.9 \\
3.0 \\
3.1 \\
3.1 \\
3.2 \\
3.3 \\
3.3 \\
3.4 \\
3.5 \\
3.6 \\
3.6 \\
3.7 \\
3.8 \\
3.8 \\
3.9\end{array}$ & $\begin{array}{l}3.0 \\
3.1 \\
3.2 \\
3.3 \\
3.4 \\
3.5 \\
3.6 \\
3.7 \\
3.8 \\
3.9 \\
4.0 \\
4.1 \\
4.2 \\
4.3 \\
4.4 \\
4.5 \\
4.6 \\
4.7 \\
4.8 \\
4.9 \\
5.0 \\
5.1 \\
5.2 \\
5.3 \\
5.4 \\
5.5 \\
5.6 \\
5.7 \\
5.8\end{array}$ & $\begin{array}{l}4.0 \\
4.1 \\
4.3 \\
4.4 \\
4.5 \\
4.7 \\
4.8 \\
4.9 \\
5.1 \\
5.2 \\
5.3 \\
5.5 \\
5.6 \\
5.7 \\
5.9 \\
6.0 \\
6.1 \\
6.3 \\
6.4 \\
6.5 \\
6.6 \\
6.8 \\
6.9 \\
7.0 \\
7.2 \\
7.3 \\
7.4 \\
7.6 \\
7.7\end{array}$ \\
\hline
\end{tabular}

$\underline{I} \mathrm{~S}_{r} / 0 \mathrm{~W}_{\mathrm{r}}=$

2/Recorded highest water level ( $\left.0 \mathrm{~W}_{\max }\right)$. 
Table 4.--Water-level adjustments, in feet, for use with index we 11 Brewster BMW-21 (located in zone B)

\begin{tabular}{|c|c|c|c|c|c|}
\hline $\begin{array}{l}\text { Measured water } \\
\text { level (OW }) \\
\text { (in feet below } \\
\text { land surface) }\end{array}$ & $\frac{\text { Zone A }}{(2 / 3) \underline{1 /}}$ & $\frac{\text { Zone B }}{(3 / 3) \underline{1 j}}$ & $\begin{array}{l}\text { Measured water } \\
\left.\text { level (OW }{ }_{c}\right) \\
\text { (in feet below } \\
\text { land surface) }\end{array}$ & $\begin{array}{l}\text { Zone A } \\
(2 / 3) 1 /\end{array}$ & $\frac{\text { Zone B }}{(3 / 3) \underline{1 /}}$ \\
\hline $\begin{array}{l}6.92 / \\
7.0 \\
7.1 \\
7.2 \\
7.3 \\
7.4 \\
7.5 \\
7.6 \\
7.7 \\
7.8 \\
7.9 \\
8.0 \\
8.1 \\
8.2 \\
8.3 \\
8.4 \\
8.5 \\
8.6 \\
8.7 \\
8.8 \\
8.9 \\
9.0 \\
9.1 \\
9.2 \\
9.3 \\
9.4 \\
9.5 \\
9.6 \\
9.7 \\
9.8 \\
9.9 \\
10.0\end{array}$ & $\begin{array}{l}0.0 \\
.1 \\
.1 \\
.2 \\
.3 \\
.3 \\
.4 \\
.5 \\
.5 \\
.6 \\
.7 \\
.7 \\
.8 \\
.9 \\
.9 \\
1.0 \\
1.1 \\
1.1 \\
1.2 \\
1.3 \\
1.3 \\
1.4 \\
1.5 \\
1.5 \\
1.6 \\
1.7 \\
1.7 \\
1.8 \\
1.9 \\
1.9 \\
2.0 \\
2.1\end{array}$ & $\begin{array}{l}0.0 \\
.1 \\
.2 \\
.3 \\
.4 \\
.5 \\
.6 \\
.7 \\
.8 \\
.9 \\
1.0 \\
1.1 \\
1.2 \\
1.3 \\
1.4 \\
1.5 \\
1.6 \\
1.7 \\
1.8 \\
1.9 \\
2.0 \\
2.1 \\
2.2 \\
2.3 \\
2.4 \\
2.5 \\
2.6 \\
2.7 \\
2.8 \\
2.9 \\
3.0 \\
3.1\end{array}$ & $\begin{array}{l}10.1 \\
10.2 \\
10.3 \\
10.4 \\
10.5 \\
10.6 \\
10.7 \\
10.8 \\
10.9 \\
11.0 \\
11.1 \\
11.2 \\
11.3 \\
11.4 \\
11.5 \\
11.6 \\
11.7 \\
11.8 \\
11.9 \\
12.0 \\
12.1 \\
12.2 \\
12.3 \\
12.4 \\
12.5 \\
12.6 \\
12.7 \\
12.8 \\
12.9 \\
13.0 \\
13.1 \\
13.2 \\
13.3\end{array}$ & $\begin{array}{l}2.1 \\
2.2 \\
2.3 \\
2.3 \\
2.4 \\
2.5 \\
2.5 \\
2.6 \\
2.7 \\
2.7 \\
2.8 \\
2.9 \\
2.9 \\
3.0 \\
3.1 \\
3.1 \\
3.2 \\
3.3 \\
3.3 \\
3.4 \\
3.5 \\
3.6 \\
3.6 \\
3.7 \\
3.8 \\
3.8 \\
3.9 \\
4.0 \\
4.0 \\
4.1 \\
4.2 \\
4.2 \\
4.3\end{array}$ & $\begin{array}{l}3.2 \\
3.3 \\
3.4 \\
3.5 \\
3.6 \\
3.7 \\
3.8 \\
3.9 \\
4.0 \\
4.1 \\
4.2 \\
4.3 \\
4.4 \\
4.5 \\
4.6 \\
4.7 \\
4.8 \\
4.9 \\
5.0 \\
5.1 \\
5.2 \\
5.3 \\
5.4 \\
5.5 \\
5.6 \\
.5 .7 \\
5.8 \\
5.9 \\
6.0 \\
6.1 \\
6.2 \\
6.3 \\
6.4\end{array}$ \\
\hline
\end{tabular}

$\underline{1 / \mathrm{S}_{r}} / 0 \mathrm{~W}_{\mathrm{r}}=$

2/Recorded highest water level $\left(O W_{\max }\right)$. 
Table 5.--Water-level adjustments, in feet, for use with index well Chatham CGW-138 (located in zone B)

\begin{tabular}{|c|c|c|c|c|}
\hline $\begin{array}{l}\text { Measured water } \\
\text { level (OW }) \\
\text { (in feet below } \\
\text { land surface) }\end{array}$ & $\frac{\text { Zone A }}{(2 / 3) \underline{1} /}$ & $\frac{\text { Zone B }}{(3 / 3)^{1}}$ & $\frac{\text { Zone C }}{(4 / 3) \underline{1} /}$ & $\frac{\text { Zone D }}{(5 / 3) \underline{1}}$ \\
\hline $\begin{array}{l}\text { 21.12 } \\
21.2 \\
21.3 \\
21.4 \\
21.5 \\
21.6 \\
21.7 \\
21.8 \\
21.9 \\
22.0 \\
22.1 \\
22.2 \\
22.3 \\
22.4 \\
22.5 \\
22.6 \\
22.7 \\
22.8 \\
22.9 \\
23.0 \\
23.1 \\
23.2 \\
23.3 \\
23.4 \\
23.5 \\
23.6 \\
23.7 \\
23.8 \\
23.9 \\
24.0 \\
24.1 \\
24.2 \\
24.3 \\
24.4 \\
24.5 \\
24.6 \\
24.7 \\
24.8 \\
24.9 \\
\end{array}$ & $\begin{array}{l}0.0 \\
.1 \\
.1 \\
.2 \\
.3 \\
.3 \\
.4 \\
.5 \\
.5 \\
.6 \\
.7 \\
.7 \\
.8 \\
.9 \\
.9 \\
1.0 \\
1.1 \\
1.1 \\
1.2 \\
1.3 \\
1.3 \\
1.4 \\
1.5 \\
1.5 \\
1.6 \\
1.7 \\
1.7 \\
1.8 \\
1.9 \\
1.9 \\
2.0 \\
2.1 \\
2.1 \\
2.2 \\
2.3 \\
2.3 \\
2.4 \\
2.5 \\
2.5 \\
2.6 \\
2.7 \\
2.7 \\
2.8 \\
2.9 \\
2.9 \\
3.0 \\
3.1 \\
3.1 \\
3.2 \\
3.3 \\
3.3 \\
3.4\end{array}$ & $\begin{array}{r}0.0 \\
.1 \\
.2 \\
.3 \\
.4 \\
.5 \\
.6 \\
.7 \\
.8 \\
.9 \\
1.0 \\
1.1 \\
1.2 \\
1.3 \\
1.4 \\
1.5 \\
1.6 \\
1.7 \\
1.8 \\
1.9 \\
2.0 \\
2.1 \\
2.2 \\
2.3 \\
2.4 \\
2.5 \\
2.6 \\
2.7 \\
2.8 \\
2.9 \\
3.0 \\
3.1 \\
3.2 \\
3.3 \\
3.4 \\
3.5 \\
3.6 \\
3.7 \\
3.8 \\
3.9 \\
4.0 \\
4.1 \\
4.2 \\
4.3 \\
4.4 \\
4.5 \\
4.6 \\
4.7 \\
4.8 \\
4.9 \\
5.0 \\
5.1 \\
\end{array}$ & $\begin{array}{l}0.0 \\
.1 \\
.3 \\
.4 \\
.5 \\
.7 \\
.8 \\
.9 \\
1.1 \\
1.2 \\
1.3 \\
1.5 \\
1.6 \\
1.7 \\
1.9 \\
2.0 \\
2.1 \\
2.3 \\
2.4 \\
2.5 \\
2.7 \\
2.8 \\
2.9 \\
3.1 \\
3.2 \\
3.3 \\
3.5 \\
3.6 \\
3.7 \\
3.9 \\
4.0 \\
4.1 \\
4.3 \\
4.4 \\
4.5 \\
4.7 \\
4.8 \\
4.9 \\
5.1 \\
5.2 \\
5.3 \\
5.5 \\
5.6 \\
5.7 \\
5.9 \\
6.0 \\
6.1 \\
6.3 \\
6.4 \\
6.5 \\
6.6 \\
6.8\end{array}$ & $\begin{array}{l}0.0 \\
.2 \\
.3 \\
.5 \\
.7 \\
.8 \\
1.0 \\
1.2 \\
1.3 \\
1.5 \\
1.7 \\
1.8 \\
2.0 \\
2.2 \\
2.3 \\
2.5 \\
2.7 \\
2.8 \\
3.0 \\
3.2 \\
3.3 \\
3.5 \\
3.7 \\
3.8 \\
4.0 \\
4.2 \\
4.3 \\
4.5 \\
4.7 \\
4.8 \\
5.0 \\
5.2 \\
5.3 \\
5.5 \\
5.7 \\
5.8 \\
6.0 \\
6.2 \\
6.3 \\
6.5 \\
6.7 \\
6.8 \\
7.0 \\
7.2 \\
7.3 \\
7.5 \\
7.7 \\
7.8 \\
8.0 \\
8.2 \\
8.3 \\
8.5\end{array}$ \\
\hline
\end{tabular}

$I / \mathrm{S}_{r} / 0 \mathrm{~W}_{\mathrm{r}}=$

2/Recorded highest water level $\left(0 \mathrm{~W}_{\max }\right)$. 
Table 6.--Water-level adjustments,

in feet, for use with index well

Sandwich SDW-252 (located in zone A)

\begin{tabular}{|c|c|c|}
\hline $\begin{array}{l}\text { Measured water } \\
\text { leve } 1 \text { (OW }) \\
\text { (in feet below } \\
\text { land surface) }\end{array}$ & $\frac{\text { Zone A }}{(2 / 2) \underline{1 /}}$ & $\frac{\text { Zone B }}{(3 / 2)^{1 /}}$ \\
\hline $\begin{array}{l}46.02 / \\
46.1 \\
46.2 \\
46.3 \\
46.4 \\
46.5 \\
46.6 \\
46.7 \\
46.8 \\
46.9 \\
47.0 \\
47.1 \\
47.2 \\
47.3 \\
47.4 \\
47.5 \\
47.6 \\
47.7 \\
47.8 \\
47.9 \\
48.0 \\
48.1 \\
48.2\end{array}$ & $\begin{array}{r}0.0 \\
.1 \\
.2 \\
.3 \\
.4 \\
.5 \\
.6 \\
.7 \\
.8 \\
.9 \\
1.0 \\
1.1 \\
1.2 \\
1.3 \\
1.4 \\
1.5 \\
1.6 \\
1.7 \\
1.8 \\
1.9 \\
2.0 \\
2.1 \\
2.2\end{array}$ & $\begin{array}{r}0.0 \\
.1 \\
.3 \\
.4 \\
.6 \\
.7 \\
.9 \\
1.0 \\
1.2 \\
1.3 \\
1.5 \\
1.6 \\
1.8 \\
1.9 \\
2.1 \\
2.2 \\
2.4 \\
2.5 \\
2.7 \\
2.8 \\
3.0 \\
3.1 \\
3.3\end{array}$ \\
\hline
\end{tabular}


Table 7.--Water-leve 1 adjustments, in feet, for use with index well Sandwich SDW-253 (located in zone A)

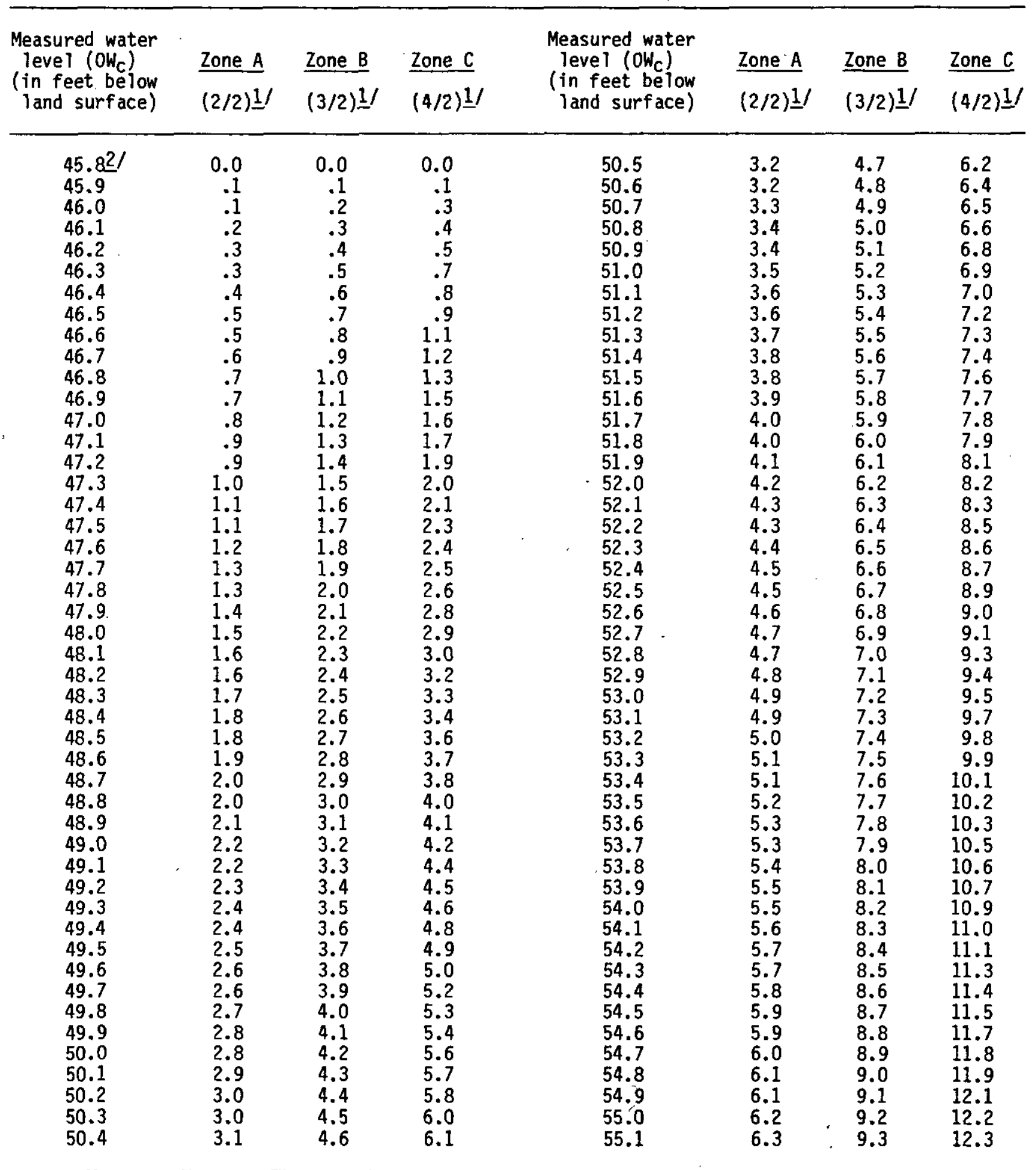

$1 / \mathrm{s}_{\mathrm{r}} / 0 \mathrm{w}_{\mathrm{r}}=$

2/Recorded highest water level (OW $\left.w_{\max }\right)$. 
Table 8. - Water-level adjustments, in feet, for use with index well Truro TSW-89 (located in zone A)

\begin{tabular}{|c|c|c|c|c|c|c|c|}
\hline $\begin{array}{l}\text { Measured water } \\
\text { level ( } \mathrm{W}_{\mathrm{C}} \text { ) } \\
\text { (in feet below } \\
\text { land surface) }\end{array}$ & $\frac{\text { Zone A }}{(2 / 2) \underline{1} \text {. }}$ & $\frac{\text { Zone B }}{(3 / 2) \underline{1} \text { ! }}$ & $\frac{\text { Zone C }}{(4 / 2) \underline{1} /}$ & $\begin{array}{l}\text { Measured water } \\
\text { level. (ow }) \\
\text { (in feet below } \\
\text { land.surface) }\end{array}$ & $\frac{\text { Zone A }}{(2 / 2) 1 /}$ & $\frac{\text { Zone B }}{(3 / 2) 1 /}$ & $\frac{\text { Zone C }}{(4 / 2) \underline{1}}$ \\
\hline $\begin{array}{l}10.42 / \\
10.5 \\
10.6 \\
10.7 \\
10.8 \\
10.9 \\
11.0 \\
11.1 \\
11.2 \\
11.3 \\
11.4 \\
11.5 \\
11.6 \\
11.7\end{array}$ & $\begin{array}{r}0.0 \\
.1 \\
.2 \\
.3 \\
.4 \\
.5 \\
.6 \\
.7 \\
.8 \\
.9 \\
1.0 \\
1.1 \\
1.2 \\
1.3\end{array}$ & $\begin{array}{r}0.0 \\
.1 \\
.3 \\
.4 \\
.6 \\
.7 \\
.9 \\
1.0 \\
1.2 \\
1.3 \\
1.5 \\
1.6 \\
1.8 \\
1.9\end{array}$ & $\begin{array}{l}0.0 \\
.2 \\
.4 \\
.6 \\
.8 \\
1.0 \\
1.2 \\
1.4 \\
1.6 \\
1.8 \\
2.0 \\
2.2 \\
2.4 \\
2.6\end{array}$ & $\begin{array}{l}11.8 \\
11.9 \\
12.0 \\
12.1 \\
12.2 \\
12.3 \\
12.4 \\
12.5 \\
12.6 \\
12.7 \\
12.8 \\
12.9 \\
13.0\end{array}$ & $\begin{array}{l}1.4 \\
1.5 \\
1.6 \\
1.7 \\
1.8 \\
1.9 \\
2.0 \\
2.1 \\
2.2 \\
2.3 \\
2.4 \\
2.5 \\
2.6\end{array}$ & $\begin{array}{l}2.1 \\
2.2 \\
2.4 \\
2.5 \\
2.7 \\
2.8 \\
3.0 \\
3.1 \\
3.3 \\
3.4 \\
3.6 \\
3.7 \\
3.9\end{array}$ & $\begin{array}{l}2.8 \\
3.0 \\
3.2 \\
3.4 \\
3.6 \\
3.8 \\
4.0 \\
4.2 \\
4.4 \\
4.6 \\
4.8 \\
5.0 \\
5.2\end{array}$ \\
\hline
\end{tabular}

$1 / \mathrm{S}_{\mathrm{r}} / 0 \mathrm{~W}_{\mathrm{r}}=$

2/Recorded highest water level $\left(0 \mathrm{~W}_{\max }\right)$.

Table 9.--Water-level adjustments, in feet, for use with index well Wellfleet WNW-17 (located in zone C)

\begin{tabular}{|c|c|c|c|c|c|c|c|}
\hline $\begin{array}{l}\text { Measured water } \\
\text { level (ow }) \\
\text { (in feet below } \\
\text { land surface) }\end{array}$ & $\frac{\text { Zone A }}{(2 / 4)^{1 /}}$ & $\frac{\text { Zone B }}{(3 / 4)^{1 /}}$ & $\frac{\text { Zone C }}{(4 / 4)^{1 /}}$ & $\begin{array}{l}\text { Measured water } \\
\text { level (OW }) \\
\text { (in feet below } \\
\text { land surface) }\end{array}$ & $\frac{\text { Zone A }}{(2 / 4) 1 /}$ & $\frac{\text { Zone B }}{(3 / 4) \underline{1} \text {; }}$ & $\frac{\text { Zone C }}{(4 / 4) \underline{1}}$ \\
\hline $\begin{array}{r}7.321 \\
7.4 \\
7.5 \\
7.6 \\
7.7 \\
7.8 \\
7.9 \\
8.0 \\
8.1 \\
8.2 \\
8.3 \\
8.4 \\
8.5 \\
8.6 \\
8.7 \\
8.8 \\
9.9 \\
9.0 \\
9.1 \\
9.2 \\
9.3 \\
9.4 \\
9.5 \\
9.6 \\
9.7 \\
9.8 \\
9.9 \\
10.0\end{array}$ & $\begin{array}{r}0.0 \\
.0 \\
.1 \\
.1 \\
.2 \\
.2 \\
.3 \\
.3 \\
.4 \\
.4 \\
.5 \\
.5 \\
.6 \\
.6 \\
.7 \\
.7 \\
.8 \\
.8 \\
.9 \\
.9 \\
1.0 \\
1.0 \\
1.1 \\
1.1 \\
1.2 \\
1.2 \\
1.3 \\
1.3\end{array}$ & $\begin{array}{l}0.0 \\
.1 \\
.1 \\
.2 \\
.3 \\
.4 \\
.4 \\
.5 \\
.6 \\
.7 \\
.7 \\
.8 \\
.9 \\
1.0 \\
1.0 \\
1.1 \\
1.2 \\
1.3 \\
1.3 \\
1.4 \\
1.5 \\
1.6 \\
1.6 \\
1.7 \\
1.8 \\
1.9 \\
1.9 \\
2.0\end{array}$ & $\begin{array}{r}0.0 \\
.1 \\
.2 \\
.3 \\
.4 \\
.5 \\
.6 \\
.7 \\
.8 \\
.9 \\
1.0 \\
.1 .1 . \\
1.2 \\
1.3 \\
1.4 \\
1.5 \\
1.6 \\
1.7 \\
1.8 \\
1.9 \\
2.0 \\
2.1 \\
2.2 \\
2.3 \\
2.4 \\
2.5 \\
2.6 \\
2.7\end{array}$ & $\begin{array}{l}10.1 \\
10.2 \\
10.3 \\
10.4 \\
10.5 \\
10.6 \\
10.7 \\
10.8 \\
10.9 \\
11.0 \\
11.1 \\
11.2 \\
11.3 \\
11.4 \\
11.5 \\
11.6 \\
11.7 \\
11.8 \\
11.9 \\
12.0 \\
12.1 \\
12.2 \\
12.3 \\
12.4 \\
12.5 \\
12.6 \\
12.7 \\
12.8\end{array}$ & $\begin{array}{l}1.4 \\
1.4 \\
1.5 \\
1.5 \\
1.6 \\
1.6 \\
1.7 \\
1.7 \\
1.8 \\
1.8 \\
1.9 \\
1.9 \\
2.0 \\
2.0 \\
2.1 \\
2.1 \\
2.2 \\
2.2 \\
2.3 \\
2.3 \\
2.4 \\
2.4 \\
2.5 \\
2.5 \\
2.6 \\
2.6 \\
2.7 \\
2.7\end{array}$ & $\begin{array}{r}2.1 \\
2.2 \\
2.2 \\
2.3 \\
2.4 \\
2.5 \\
2.5 \\
2.6 \\
2.7 \\
2.8 \\
2.8 \\
2.9 \\
3.0 \\
3.1 \\
3.1 \\
3.2 \\
3.3 \\
3.4 \\
3.4 \\
3.5 \\
.3 .6 \\
3.7 \\
3.7 \\
3.8 \\
.3 .9 \\
4.0 \\
4.0 \\
4.1\end{array}$ & $\begin{array}{l}2.8 \\
2.9 \\
3.0 \\
3.1 \\
3.2 \\
3.3 \\
3.4 \\
3.5 \\
3.6 \\
3.7 \\
3.8 \\
3.9 \\
4.0 \\
4.1 \\
4.2 \\
4.3 \\
4.4 \\
4.5 \\
4.6 \\
4.7 \\
4.8 \\
4.9 \\
5.0 \\
5.1 \\
5.2 \\
5.3 \\
5.4 \\
5.5\end{array}$ \\
\hline
\end{tabular}

$\underline{1} / \mathrm{S}_{\mathrm{r}} / 0 \mathrm{~W}_{\mathrm{r}}=$

2/Recorded highest water level $\left(0 W_{\max }\right)$. 
Water levels in short-term well AIW 306 are correlated with those in long-term observation well AIW 230, as shown in figure 4. For the period October 1975 to March 1977, the line of relationship between water levels at these two observation wells is represented by the regression equation AIW $306=0.954 \mathrm{AIW} 230+3.31$, for which the correlation coefficient is 0.985 and the standard error of estimate is 0.20 feet. Using the line of relationship in figure 4, or the above equation based on past water levels, 66 percent of the estimates of water levels in AIW 306 made from water levels in AIW 230 are expected to have an error of less than 0.20 feet. Correlation coefficient is a measure of degree of association between two variables and, in this situation, a measure of the ability to estimate water levels at one well from water levels at another well. A perfect relationship is represented by a correlation coefficient of 1.0. Within these mapped areas, 87 percent of the correlation coefficients were greater than 0.8, and 60 percent were greater than 0.9. During the period 1975-77, the water-level fluctuations on Cape Cod were greater than average, but not the greatest of record. The water-level fluctuations in the nine index wells during the 1975-77 period were exceeded in only 15 percent of a composite 145 years of total record for the nine wells.

On a basis of the annual water-level ranges measured in the nine long-term and 146 shortterm wells for the October 1975 to March 1977 period, as well as proximity to pumping centers and constant head hydrologic boundaries and aquifer transmissivity, five zones of annual groundwater level range were delineated. The zones were mapped on plates $I$ and 2 as: $A$, (water-level fluctuations 0 to 2 feet), B (2-3 feet), C (3-4 feet), D (4-5 feet), and E (5-6 feet). Generally, the water-level fluctuations in the wells were rounded up to the nearest half foot before the boundaries between zones were mapped. This rule was not followed at about 8 percent of the locations in order to maintain simple areal continuity of mapping and to recognize the weight of data at adjacent well locations. The zones mapped on plates $l$ and 2 represent annual water-level ranges and are less than the ranges for the total periods of record at the long-term observation wells.

Values of $\mathrm{S}_{\mathrm{r}} / O \mathrm{~W}_{\mathrm{r}} \cdot\left(\mathrm{OW} \mathrm{W}_{\mathrm{C}}-\mathrm{OW}_{\mathrm{max}}\right.$ ) were prepared for nine index wells selected from the 13 long-term observation wells ( 16 to 28 years of records). The values in the tables were computed by dividing the difference between the recorded maximum and minimum water levels for the total periods of record into 0.1 foot increments. The differences between the measured water level and the recorded highest water level observed in an index well is then multiplied by $\left(\mathrm{S}_{\mathrm{r}} / \mathrm{OW}_{\mathrm{r}}\right)$ to obtain the water-level adjustments for different annual ranges for that index well (tables 1-9). With these tables and the identification of the correlated index well and the annual water-level range zone which for a test site is located, the probable high water-level at the test site can be estimated.

\section{Procedure of Estimating the Probable High Water Level}

The depth to the probable high water level at any site on Cape Cod where regulations for septic-tank construction requires that the maximum ground-water level be more. than 4 feet below the bottom of the leaching field ( 7 feet below land surface if a 3 foot deep leaching field is assumed) may be estimated by the following steps (plate 3 ).

1. Measure, to the nearest one tenth of a foot, the depth to water below land surface at the site.

2. If the water level is less than 16 feet but more than 7 feet below land surface, locate the site on plate 1 or 2 and determine the appropriate zone and the index well.

3. Determine the maximum depth to water-level adjustment value in tables 1-9 which represents the appropriate index well and the zone.

4. Subtract the maximum depth to water-level adjustiment value from the measured depth to water. If the result is greater than 7 feet, the water table is not expected to rise to within 7 feet of land surface. If, however, the value is less than 7 feet, proceed to step 5 .

5. Determine the depth to water in the appropriate index well for the month in which the depth to water was measured at the site (or previous month if the current data are not available). (The U.S. Geological Survey reports the index-well measurements to the Regional Engineers of the Massachusetts Department of Environmental Quality Engineering monthly.) Apply the same table as used in item 3 and use the reported depth to water in the index well and the appropriate zone where the site is located to determine the depth to water-level adjustment value from the table. 


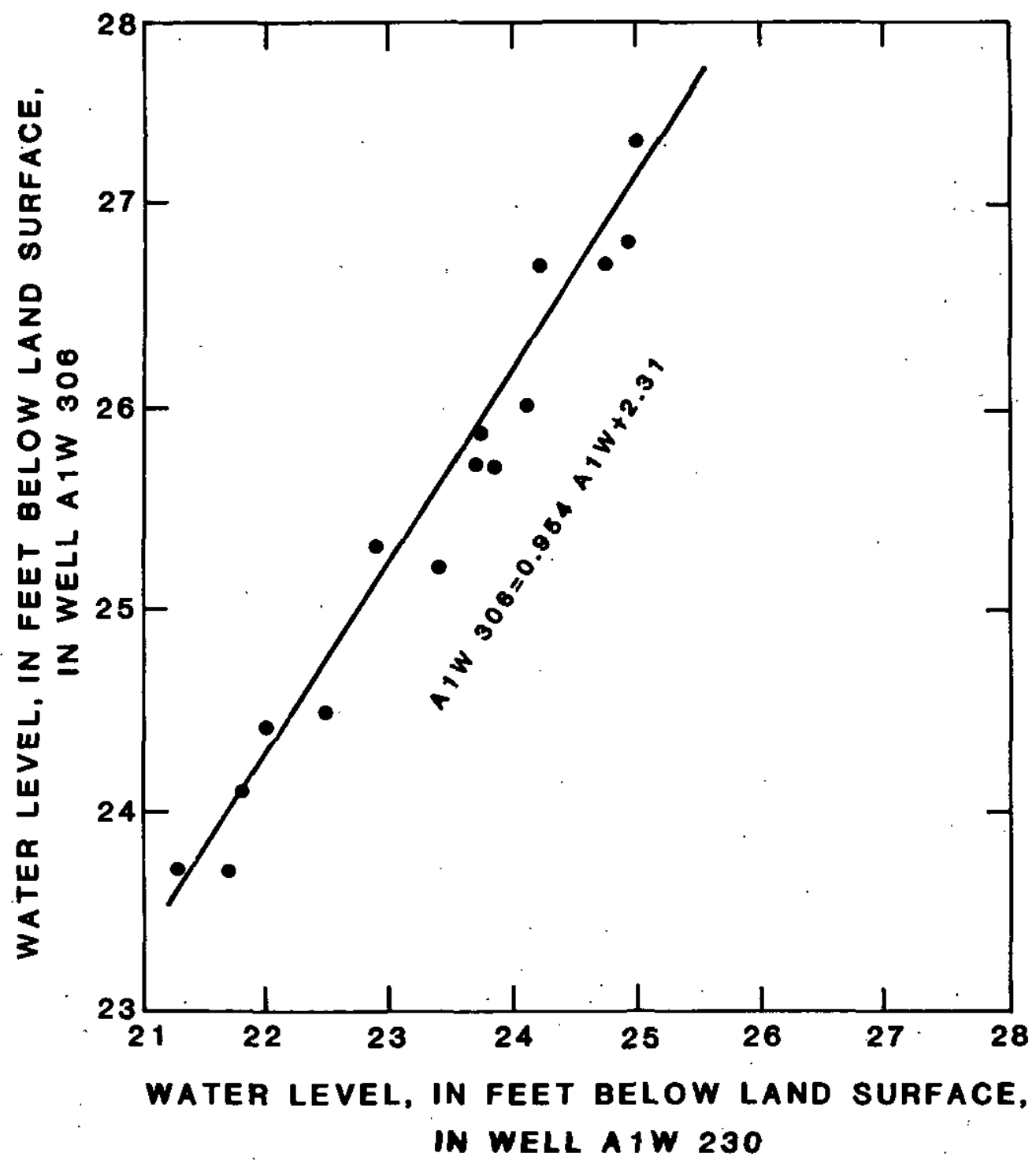

Figure 4.-- Correlation of water levels in two observation wells 
6. Subtract the determined depth to water-level adjustment value from the measured depth to water to obtain the estimate of depth to the probable high water level. If the estimate is greater than 7 feet below land surface, the water level is not expected to rise to within 4 feet of the bottom of a leach field installed at the site. If the estimate is less than 7 feet, the water table is expected to rise to within 4 feet of the bottom of a leach field installed at the site.

Because the locations of the boundaries between the areas represented by the index wells are somewhat arbitrary, it is suggested that, when the site being evaluated is within 1,000 feet of such a boundary, estimates should be calculated from both index wells. The higher of the two ground-water levels calculated would be less likely to be exceeded.

The water level for the month in which the site testing was done should be used in step 5. However, the water level reported for the index well for the month previous to the month in which the site was tested may be used, provided that 0.25 feet is subtracted from the depth to the water level for each week or fraction thereof between the date of the site test and the end of the previous month. For example, if an estimate for a site test made on the 8th of August $(8 / 7=1.14)$ should be adjusted by subtracting 0.3 feet from the reported July water level for the index well. This adjustment need only be applied when the test site is measured in the months of May, June, July, August, September, or October. The adjustment is based on the recorded maximum water-level decline of 0.99 feet over a Imonth period in all nine index wells and the observation that ground-water levels on Cape Cod generally decline from May through October.

\section{An Example}

Assume, step 1-plate 4, that a septic system is proposed for a site near the intersection of Higgins Crowell Road and Route 28 in West Yarmouth (plate 1) and that at that site a measurement of depth to water was 10.2 feet below land surface in August 1978. From the map (plate 1), the index well for the location of the site was identified as TSW-89 and the Zone was Zone B.

From the bottom line of table 8 for Zone $B$, the maximum depth to water-level adjustment value was 3.9 feet. Subtracting this value ( 3.9 feet) from the measured depth to water at the site (10.2 feet), the estimate of the probable high water level is 6.3 feet below land surface.

If the estimate is less than 7 feet, then determine the depth of water in the index well TSW-89 at the time the depth to water was measured at the site. However, for this example, only the July water level of index wells reported by the U.S. Geological Survey is available. The reported July water level in index well TSW-89 was $12.08 \mathrm{feet}$, rounded to 12.1 feet, below land surface (table 10).

In table 8 , the depth to water-level adjustment value for zone B and depth to water of 12.1 feet is 2.5 feet.

Subtract 2.5 feet from the measured depth of water ( 10.2 feet) to estimate the probable depth to high water level at 7.7 feet which is more than 7 feet below land surface, therefore, in this example, the depth to water is not expected to rise above 7 feet. If the estimated water level were less than 7 feet below land surface, then the water level probably would be less than 4 feet below the bottom of a leach field.

\section{SUMMARY AND CONCLUSIONS}

The procedure described in this report can be used to estimate the probable high water level in sand and gravel areas of Cape Cod. It cannot be applied in areas where ground water is perched or where poorly permeable layers of silt and clay or till are present. Sites should be inspected for indications of perched ground water and silt and clay or till before attempting to estimate the water levels. Some parts of Cape Cod on platel show no pattern for annual water-level range. In these locations, soil conditions are unsuitable for water level estimation.

An analysis of the reliability of the estimation at 49 observation wells during the period from October 1975 to March 1980 was made (fig. 5). The 49 observation wells are part of the 146 short-term (October 1975 to March 1977) observation-well network used in preparation of plates 1 and 2. 
Table 10.-Example of U.S. Geological Survey monthly

PROVISIONAL summary of ground-water levels in Massachusetts; July 1978 PROVISIONAL

\begin{tabular}{|c|c|c|c|c|c|c|c|c|c|c|c|}
\hline & & & $\frac{\text { level }}{\text { Day }}$ & \multicolumn{2}{|c|}{ Net change } & & \multicolumn{4}{|c|}{ Extremes for July } \\
\hline $\begin{array}{l}\text { cton } 158 \\
\text { ndover } 26 \\
\text { ndover } 462 \\
\text { ttleboro } 83 \\
\text { arnstable } 230 \\
\text { arnstable } 247 \\
\text { illerica } 363 \\
\text { oston } 925 . \\
\text { ourne } 198 \\
\text { rewster } 21\end{array}$ & - & $\begin{array}{c}\begin{array}{c}\text { of } \\
\text { record } \\
\text { (mon-yr) }\end{array} \\
1-65 \\
5-62 \\
11-68 \\
6-64 \\
12-57 \\
11-62 \\
5-62 \\
2-60 \\
11-62 \\
10-62\end{array}$ & $\begin{array}{l}\text { Feet } \\
\text { below } \\
\text { Isd } \\
18.98 \\
12.01 \\
14.76 \\
4.53 \\
22.65 \\
22.53 \\
9.19 \\
21.52 \\
32.00 \\
8.96\end{array}$ & $\begin{array}{l}27 \\
27 \\
27 \\
25 \\
27 \\
27 \\
27 \\
27 \\
27 \\
27\end{array}$ & $\begin{array}{l}\text { month } \\
(\mathrm{ft}) \\
-0.59 \\
-.81 \\
-.52 \\
-.43 \\
-.47 \\
-1.08 \\
-1.87 \\
+.05 \\
-.47 \\
-.06\end{array}$ & $\begin{array}{l}\begin{array}{c}\text { Year } \\
\text { (ft) }\end{array} \\
-0.09 \\
+.09 \\
+.04 \\
-.05 \\
(1) \\
+1.32 \\
+.44 \\
+.69 \\
+.19 \\
+1.83\end{array}$ & $\begin{array}{c}\begin{array}{c}\text { from July } \\
\text { average } \\
\text { (ft) }\end{array} \\
-0.22 \\
-.22 \\
-.14 \\
-.13 \\
+.91 \\
+1.59 \\
-.23 \\
+1.56 \\
+1.27 \\
+.46\end{array}$ & $\begin{array}{r}\begin{array}{r}\text { High } \\
(\mathrm{ft})\end{array} \\
17.58 \\
10.02 \\
13.60 \\
3.70 \\
21.78 \\
21.52 \\
6.69 \\
21.25 \\
31.50 \\
7.12\end{array}$ & $\begin{array}{l}\text { Date } \\
\text { (yr) }\end{array}$ & $\begin{array}{l}\begin{array}{c}\text { Low } \\
(\mathrm{ft})\end{array} \\
20.62 \\
12.48 \\
15.15 \\
4.90 \\
25.46 \\
27.82 \\
10.34 \\
25.28 \\
35.51 \\
12.97\end{array}$ & 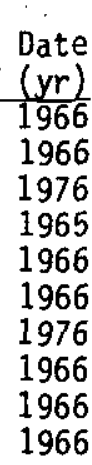 \\
\hline $\begin{array}{l}\text { ewster } 22 \\
\text { hbridge } 667 \\
\text { atham } 138 \\
\text { kbury } 79 \\
\text { kbury } 80 \\
\text { t Bridgewater } \\
11 \text { River } 67 \\
\text { Imouth } 5 \\
\text { kborough } 3 \\
\text { etown } 23\end{array}$ & 30 & $\begin{array}{r}11-62 \\
2-45 \\
11-62 \\
1-65 \\
4-65 \\
7-58 \\
4-48 \\
8-50 \\
1-65 \\
.6-64\end{array}$ & $\begin{array}{r}29.91 \\
7.32 \\
22.74 \\
8.85 \\
22.44 \\
10.75 \\
8.99 \\
4.42 \\
19.36 \\
13.19\end{array}$ & $\begin{array}{l}27 \\
26 \\
27 \\
24 \\
24 \\
24 \\
24 \\
27 \\
24 \\
25\end{array}$ & $\begin{array}{r}-.16 \\
-1.01 \\
-.22 \\
-.43 \\
-.45 \\
-2.37 \\
-.69 \\
-.34 \\
-.74 \\
-.58\end{array}$ & $\begin{array}{l}+1.69 \\
+2.16 \\
+1.04 \\
+.15 \\
+.20 \\
-.31 \\
-.38 \\
+.03 \\
+.07 \\
+.13\end{array}$ & $\begin{array}{r}+.53 \\
+2.13 \\
+.80 \\
+.42 \\
+.43 \\
.00 \\
-.19 \\
+.12 \\
-.11 \\
+.19\end{array}$ & $\begin{array}{r}21 \\
8 \\
22 \\
7 \\
7 \\
3 \\
18\end{array}$ & & $\begin{array}{l}53 \\
20 \\
25 \\
10 \\
23 \\
13\end{array}$ & $\begin{array}{l}1966 \\
1965 \\
1965 \\
1965 \\
1965 \\
1976 \\
1952 \\
1952 \\
1965 \\
1966\end{array}$ \\
\hline $\begin{array}{l}76 \\
\text { ick } 1 \\
\text { i } 11123 \\
\text { ille } 14 \\
\text { gton } 104 \\
14 \\
\text { ee } 13 \\
\text { eborough } 82 \\
\text { gue } 5 \\
\text { edford } 116\end{array}$ & & $\begin{array}{r}6-64 \\
1-65 \\
10-60 \\
6-64 \\
1-65 \\
5-39 \\
11-62 \\
1-65 \\
10-42 \\
6-64\end{array}$ & $\begin{array}{r}5.19 \\
15.63 \\
12.11 \\
15.23 \\
3.52 \\
13.00 \\
29.84 \\
13.05 \\
3.44 \\
4.35\end{array}$ & $\begin{array}{l}24 \\
24 \\
26 \\
25 \\
26 \\
26 \\
27 \\
25 \\
24 \\
25\end{array}$ & $\begin{array}{r}-.30 \\
-.95 \\
-1.51 \\
-1.61 \\
-.19 \\
-.64 \\
+.09 \\
-3.41 \\
-.97 \\
+.02\end{array}$ & $\begin{array}{l}-.18 \\
+.31 \\
+.47 \\
+.25 \\
-.14 \\
+.54 \\
+.88 \\
-1.67 \\
-.18 \\
+.08\end{array}$ & $\begin{array}{r}+.0 \\
-.0 \\
+.5 \\
+1.1 \\
-.0 \\
+.5 \\
+1.1 \\
-.5 \\
+.6 \\
+.1\end{array}$ & & & & $\begin{array}{l}1976 \\
1965 \\
1976 \\
1966 \\
1966 \\
1965 \\
1966 \\
1976 \\
1965 \\
1964\end{array}$ \\
\hline $\begin{array}{l}\text { lewbury } 27 \\
\text { lorthborough } 38 \\
\text { lorthbridge } 1 \\
\text { lorfolk } 27 \\
\text { lorton } 37 \\
\text { range } 5 \\
\text { tis } 7 \\
\text { ittsfield } 51 \\
\text { lymouth } 22 \\
\text { ilisbury } 9\end{array}$ & & $\begin{array}{r}2-65 \\
8-62 \\
1-65 \\
1-65 \\
6-64 \\
12-57 \\
1-65 \\
8-63 \\
10-56 \\
8-60\end{array}$ & $\begin{array}{r}9 . \\
8.0 \\
2 . \\
6 . \\
9 . \\
6 . \\
9 . \\
17 . \\
22 . \\
6 .\end{array}$ & $\begin{array}{l}26 \\
24 \\
24 \\
24 \\
25 \\
24 \\
25 \\
25 \\
25 \\
26\end{array}$ & $\begin{array}{r}-1.32 \\
-.24 \\
-.56 \\
-1.27 \\
-.56 \\
-.90 \\
-2.05 \\
-. .55 \\
-1.24\end{array}$ & $\begin{array}{r}+.73 \\
+.24 \\
+.29 \\
+.10 \\
-.72 \\
-.64 \\
+.44 \\
-.90 \\
+1.30 \\
+.25\end{array}$ & $\begin{array}{r}+.12 \\
-.30 \\
+.30 \\
+.02 \\
-.51 \\
-.42 \\
-.44 \\
-1.13 \\
+1.18 \\
-.21\end{array}$ & & & $\begin{array}{r}9 \\
4 \\
7 \\
10 \\
7 \\
9 \\
21 \\
28 \\
7\end{array}$ & $\begin{array}{l}19 \\
19 \\
19 \\
19 \\
10 \\
10 \\
10 \\
19 \\
10\end{array}$ \\
\hline $\begin{array}{l}\text { andwich } 252 \\
\text { andwich } 253 \\
\text { eekonk } 275 \\
\text { aunton } 337 \\
\text { ruro 1 } \\
\text { ruro } 89 . \\
\text { lakefield } 38 \\
\text { lare } 43 \\
\text { lareham } 51 \\
\text { layland } 2\end{array}$ & & $\begin{array}{r}11-62 \\
11-62 \\
6-64 \\
6-64 \\
8-50 \\
9-62 \\
1-65 \\
1-65 \\
12-59 \\
1-65\end{array}$ & $\begin{array}{r}46 . \\
46 . \\
7 . \\
9 . \\
11 . \\
12 . \\
8 . \\
9 . \\
7 . \\
16 .\end{array}$ & $\begin{array}{l}27 \\
27 \\
25 \\
25 \\
27 \\
27 \\
26 \\
24 \\
25 \\
27\end{array}$ & $\begin{array}{l}-.42 \\
-.03 \\
-.24 \\
-.48 \\
-.15 \\
-.28 \\
-.20\end{array}$ & $\begin{array}{r}+.33 \\
+2.05 \\
+.12 \\
-.17 \\
(1) \\
+.24 \\
-.43 \\
+.36 \\
+.06 \\
+.10\end{array}$ & $\begin{array}{r}+.47 \\
+2.67 \\
.0 \\
-.18 \\
.00 \\
+.16 \\
-.22 \\
+.13 \\
+.22 \\
-.32\end{array}$ & $\begin{array}{r}46 \\
45 \\
6\end{array}$ & & $\begin{array}{l}11 \\
12\end{array}$ & $\begin{array}{l}1966 \\
1966 \\
1974 \\
1976 \\
1954 \\
1965 \\
1966 \\
1966 \\
1966 \\
1976\end{array}$ \\
\hline $\begin{array}{l}\text { ebster } 1 \\
\text { ellfleet } 17 \\
\text { enham } 76\end{array}$ & & $\begin{array}{r}10-58 \\
11-62 \\
1-65\end{array}$ & 95 & $\begin{array}{l}25 \\
27\end{array}$ & $\begin{array}{l}-.9 \\
-.3\end{array}$ & $\begin{array}{r}+1.21 \\
-.76 \\
\end{array}$ & $\begin{array}{r}-.47 \\
+.44\end{array}$ & $\begin{array}{l}1.86 \\
7.77 \\
2.69\end{array}$ & & $\begin{array}{r}11.17 \\
: 4.22\end{array}$ & $\begin{array}{l}1964 \\
1966 \\
1976 \\
\end{array}$ \\
\hline
\end{tabular}

Data not available. 


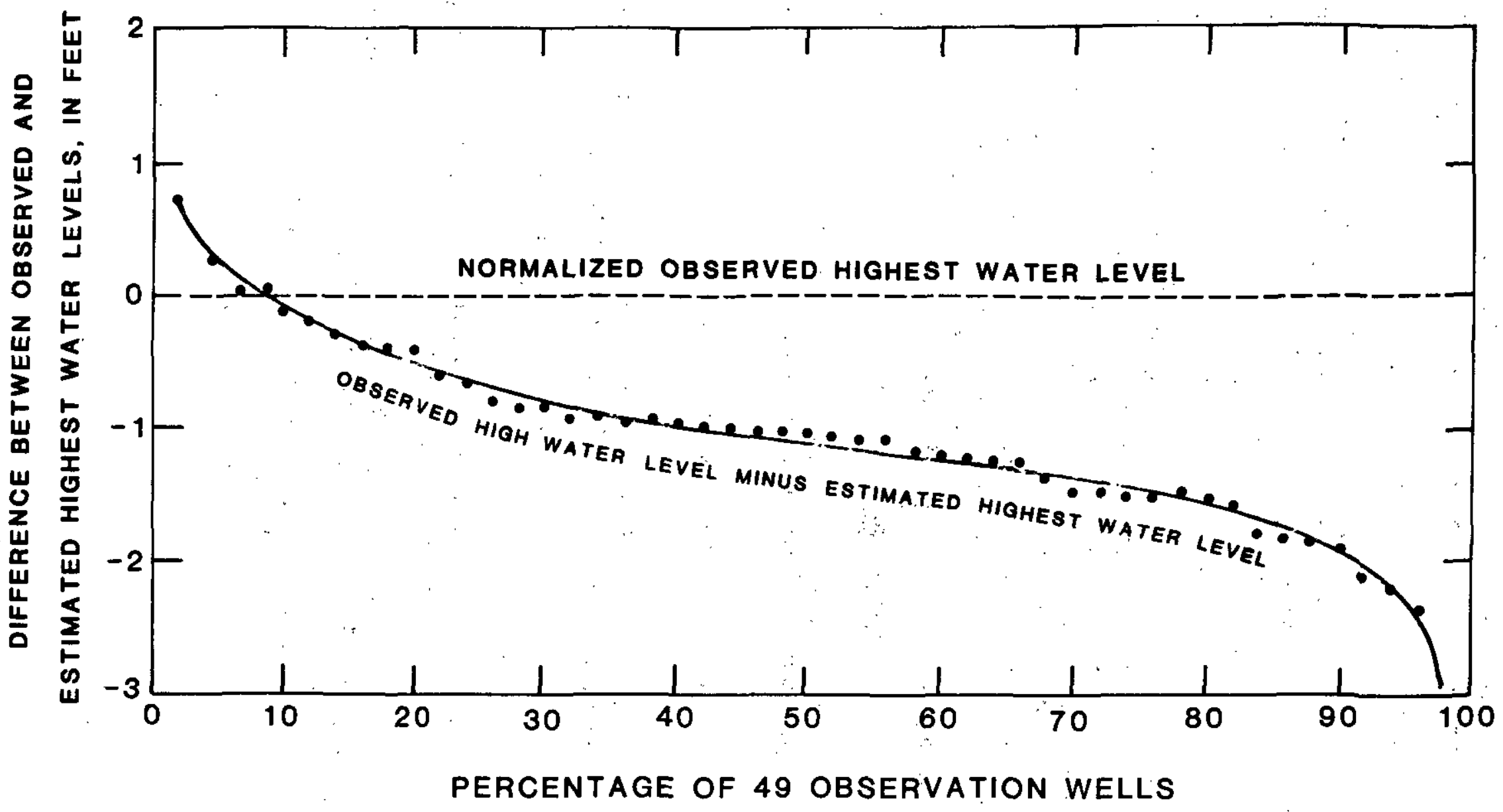

Figure 5.-- Difference between observed and estimated highest water levels at 49 sites 
Ninety-two percent of the estimates exceeded the observed maximum water levels. Only 8 percent of the estimates were exceeded by the observed water levels and by a maximum of about $3 / 4$ foot. Ninety percent of the estimates were within about 2.4 feet higher than the observed highest water levels. This indicates that the estimate errors are small and acceptable and the estimates are conservative.

Timely accessibility to current ground-water level data is required for water-level estimation. Since January 1978, monthly water-level summaries for long-term observation wells (including the index wells on Cape Cod) in Massachusetts have been sent to the five Department of Environmental Quality Engineering Regional Engineers for their use and for use by town boards of health who are responsible for issuing permits for septic systems.

The estimation of probable high water level is dependent on availability of current water-level data from a network of index wells. Collection and reporting of water-level data from the network must be continued if the estimation is needed. Owing to changing uses and ownership of land, observation wells occasionally become unavailable or are destroyed and must be replaced. A network of about 50 observation wells on Cape Cod has been identified from the 146 project observation wells and is maintained by Barnstable County and the National Park Service in cooperation with the U.S. Geological Survey. These wells now have only a few years of record, but are a contingency source of replacement wells for those used as index wells as described in this report. Additional water-level records and adjustments account for changes of pumping rates and locations as well as drainage modification would be required to increase the accuracy of the water-level estimation.

\section{SELECTED BIBLIOGRAPHY}

Commonwealth of Massachusetts, 1977, Minimum requirements for the subsurface disposal of sanitary sewage: Department of Environmental Quality Engineering Environmental Code, Title 5, $48 \mathrm{p}$.

Heath, R. C., 1976, Design of ground-water level observation-well programs: Ground Water, v. 14, no. 2 , p. $71-77$.

LeBlanc, D. R., and Guswa, J. H., 1977, Water-table map of Cape Cod, Massachusetts, May 23-27, 1976: U.S. Geological Survey Open-File Report 77-419, 2 sheets.

Marie, J. R., 1976, Preliminary evaluation of the ground-water data network in Indiana: U.S. Geological Survey Water-Resources Investigations 76-24, $44 \mathrm{p}$.

Mavesky, Anthony, 1976, Ground-water levels in Massachusetts 1936-74: U.S. Geological Survey Open-File Report, Massachusetts Hydrologic-Data Report 17, 107 p.

Moss, M. E., Lettenmaier, D. P., and Wood, E. F., 1978, On the design of hydrologic-data networks: American Geophysical Union Transactions, v. 59, no. 8, p. 772-775.

Tasker, G. D., and Guswa, J. H., 1978, Application of a mathematical model to estimate water levels: Ground Water, v. 16, no. 1, p. 18-21.

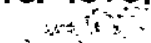

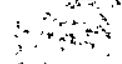



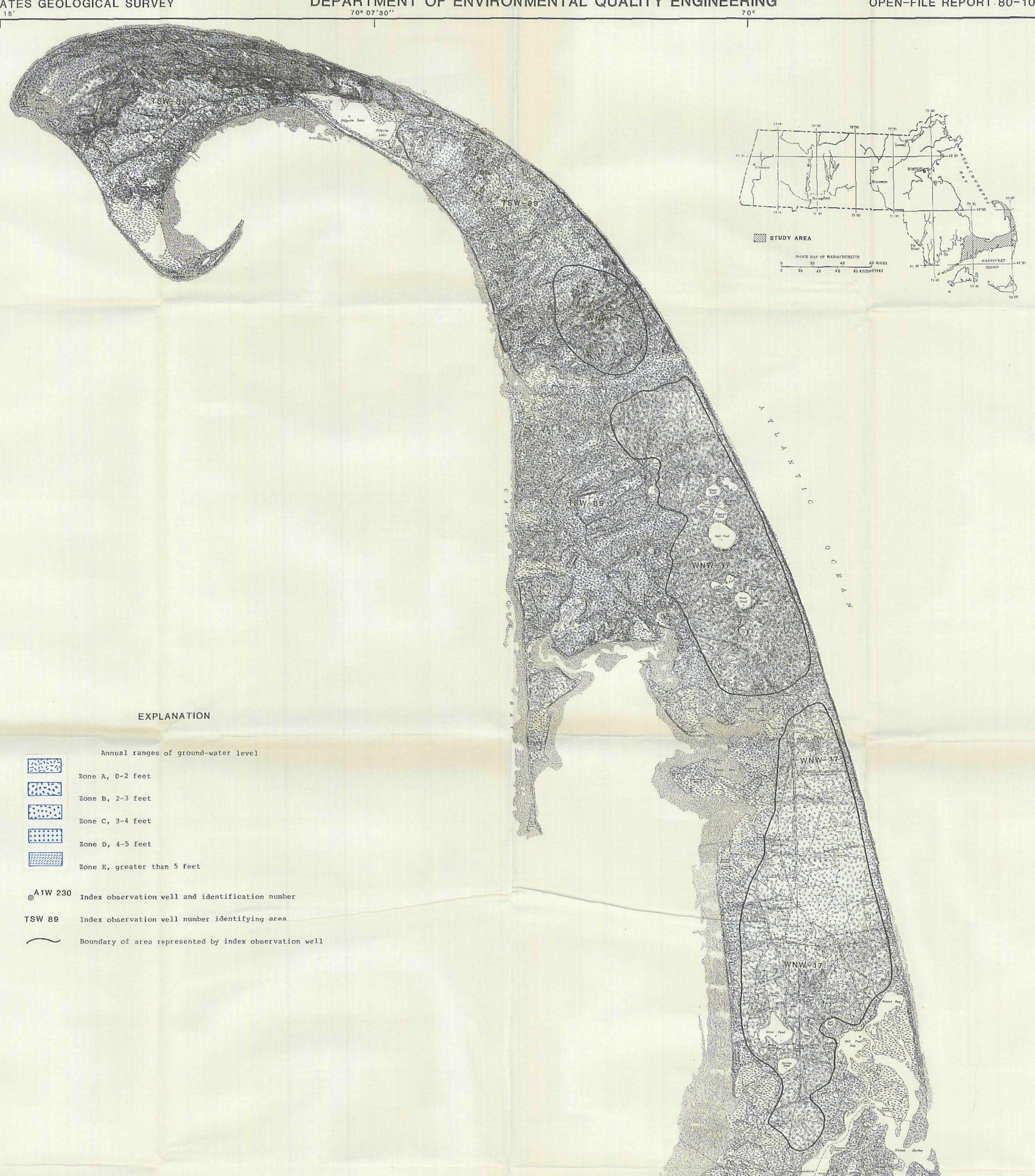

SW 89 Index observation vell number identitifying area

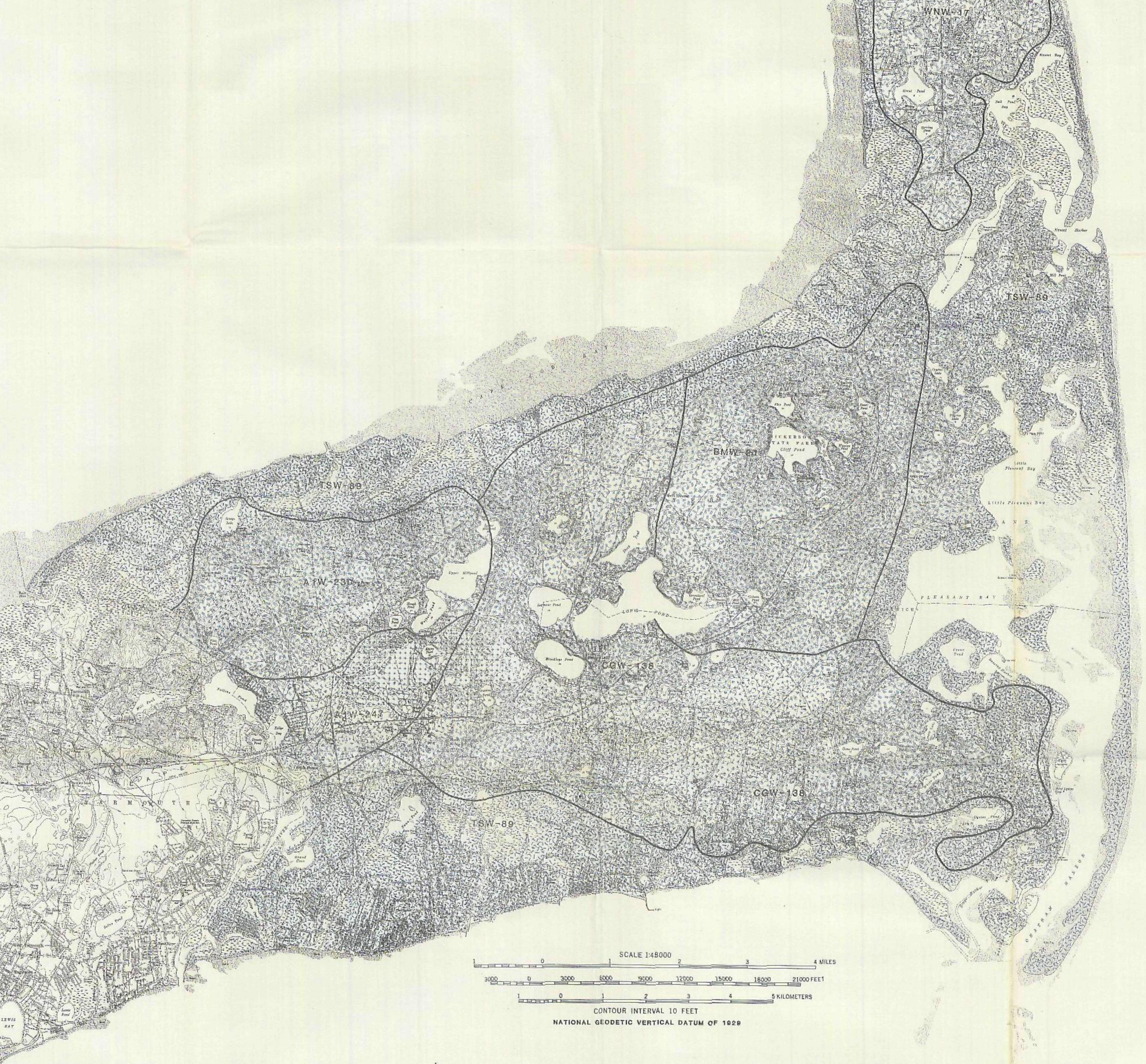
$\left(\begin{array}{c}u=w \\ n \rightarrow t\end{array}\right)$ 


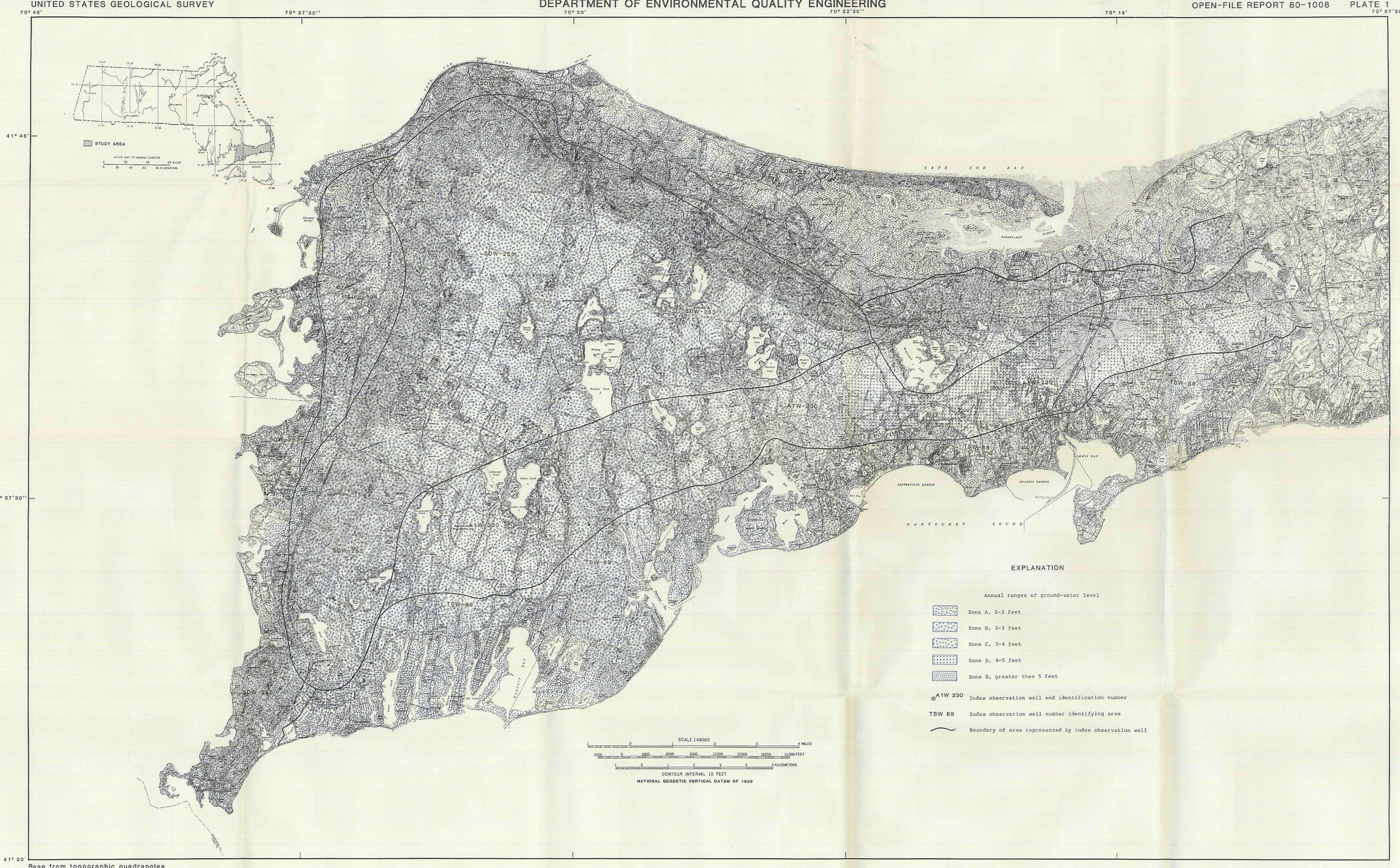




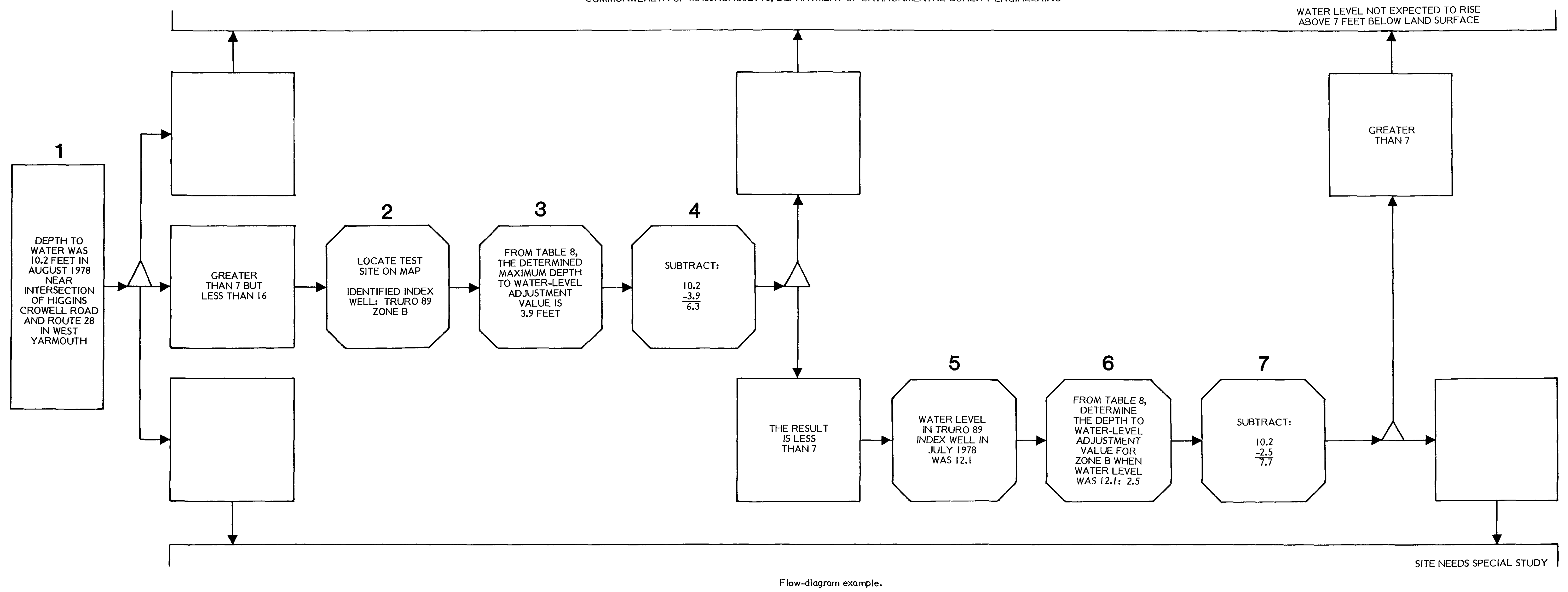




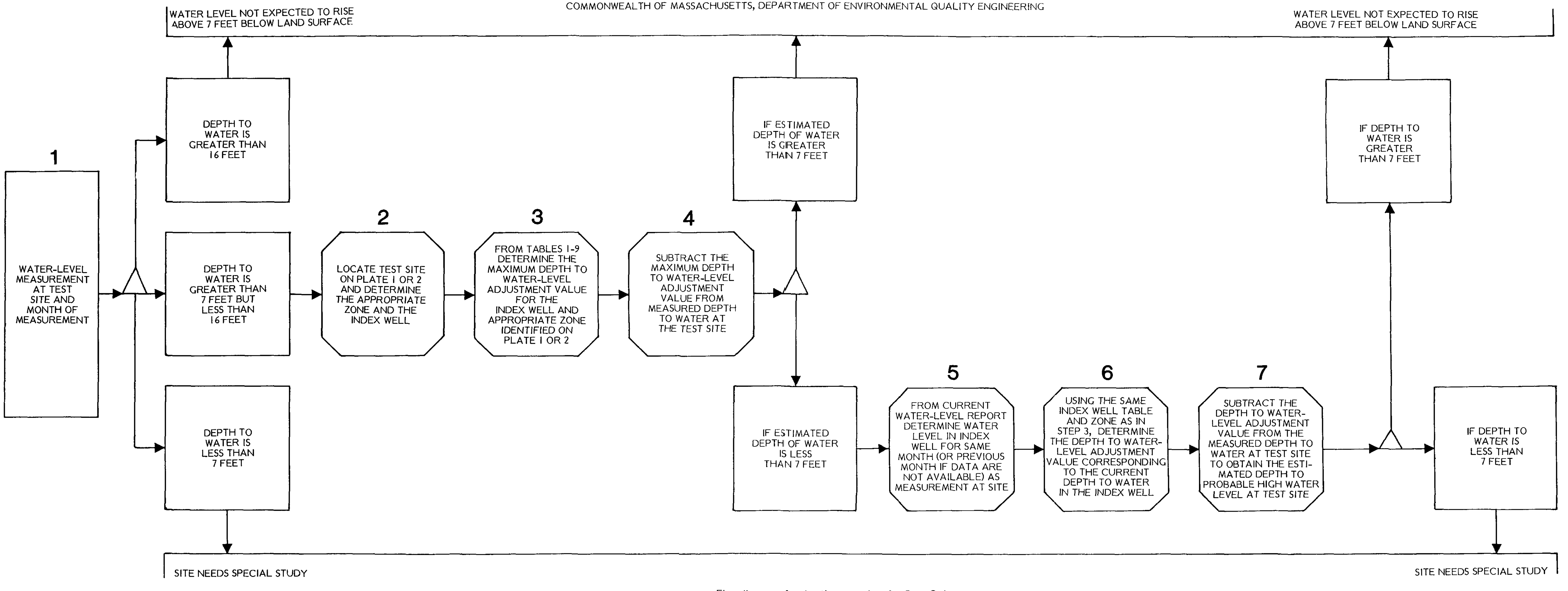


